\title{
Topology optimization of constrained layer damping on plates using Method of Moving Asymptote (MMA) approach
}

\author{
Zheng Ling ${ }^{\mathrm{a}, *}$, Xie Ronglu ${ }^{\mathrm{a}}$, Wang $\mathrm{Yi}^{\mathrm{a}}$ and Adel El-Sabbagh ${ }^{\mathrm{b}}$ \\ ${ }^{a}$ State Key Laboratory of Mechanical Transmission, Chongqing University, Chongqing 400044, P.R. China \\ ${ }^{\mathrm{b}}$ Design and Production Engineering Department, Faculty of Engineering, Ain Shams University, Cairo, Egypt
}

Received 10 February 2010

Revised 30 May 2010

\begin{abstract}
Damping treatments have been extensively used as a powerful means to damp out structural resonant vibrations. Usually, damping materials are fully covered on the surface of plates. The drawbacks of this conventional treatment are also obvious due to an added mass and excess material consumption. Therefore, it is not always economical and effective from an optimization design view. In this paper, a topology optimization approach is presented to maximize the modal damping ratio of the plate with constrained layer damping treatment. The governing equation of motion of the plate is derived on the basis of energy approach. A finite element model to describe dynamic performances of the plate is developed and used along with an optimization algorithm in order to determine the optimal topologies of constrained layer damping layout on the plate. The damping of visco-elastic layer is modeled by the complex modulus formula. Considering the vibration and energy dissipation mode of the plate with constrained layer damping treatment, damping material density and volume factor are considered as design variable and constraint respectively. Meantime, the modal damping ratio of the plate is assigned as the objective function in the topology optimization approach. The sensitivity of modal damping ratio to design variable is further derived and Method of Moving Asymptote (MMA) is adopted to search the optimized topologies of constrained layer damping layout on the plate. Numerical examples are used to demonstrate the effectiveness of the proposed topology optimization approach. The results show that vibration energy dissipation of the plates can be enhanced by the optimal constrained layer damping layout. This optimal technology can be further extended to vibration attenuation of sandwich cylindrical shells which constitute the major building block of many critical structures such as cabins of aircrafts, hulls of submarines and bodies of rockets and missiles as an invaluable design tool.
\end{abstract}

Keywords: Topology optimization, sensitivity, Method of Moving Asymptote

\section{Introduction}

Vibration and noise control of flexible structures through passive damping treatments is recognized as an effective and successful technique to solve resonant noise and vibration problems [1]. Such treatments are usually classified in two categories, unconstrained "free" layer damping and Constrained Layer Damping (CLD). The unconstrained layer damping configuration consists of a damping material, usually of visco-elastic type, bonded to the outer surfaces of the vibrating structure. On the other hand, applying a stiff layer to the outer surface of the unconstrained damping layer constrains its motion and results in a Constrained Layer Damping (CLD). The CLD treatment provides considerably more damping effect than the free visco-elastic treatment in spite of its relative complexity. Recently,

*Corresponding author. Tel.: +86 13883973842; E-mail: zling@cqu.edu.cn. 
the introduction of smart materials, such as piezo-electric materials and the advancement in computing technology has led to significant development in the control of flexible structures by presenting the concept of Active Constrained Layer Damping (ACLD). The ACLD technique has a significant advantage of attenuating low frequency vibrations. However, ACLD treatments are usually more complicated and they cost a lot more than Passive Constrained Layer Damping (PCLD) treatments.

Among the first to study visco-elastic damping, Kerwin [2] developed a simplified theory to calculate the loss factor of a plate with constrained layer damping. The theory is based on relating the shear strain of the damping layer to the transverse motion of the structure. DiTaranto and Blasingame [3] extended Kerwin's work by accounting for the extensional deformations in the visco-elastic layer and obtained composite loss factors for three and five layer beams as a function of frequency. DiTaranto [4] developed the sixth-order differential equations of motion for beams in terms of axial displacements. In the same manner, Mead and Markus [5] derived sixth-order equation of motion for beams in terms of transverse displacements. DiTaranto [4] and Mead [5] all extended Kerwin's work and examined various possible boundary conditions that can be encountered in practice. Katica [6] investigated multi-frequency vibrations of a system of two isotropic circular plates interconnected by a visco-elastic layer that has non-linear characteristics.A series of the amplitude - frequency and phase - frequency curves of the two frequency like vibration regimes are presented. These curves present the evolution of the first asymptotic approximation of solutions for different non-linear harmonics obtained by changing external excitation frequencies through discrete as well as continuous values.Therefore, transversal oscillations of the sandwich double circular plate system with visco-non-linear elastic layer are approximately solved for various initial conditions and external excitation.

Plunkett and Lee [7] were the first to study optimization for constrained layer damping beams. They presented a method to increase the energy dissipation in the structure by placing segmented constraining layers of optimum length on an unconstrained beam. Alam and Asnani [8] derived the variational equations of motion for general multilayered plate with alternating visco-elastic and stiff layers of orthotropic material, they concluded that damping of flexural modes may be optimized by proper selection of the thickness ratio of the visco-elastic layer to that of the constraining layer. Johnson and Kienholz [9] introduced a practical analysis to predict the damping characteristics of damped structures by the Modal Strain Energy (MSE) method, using the finite element analysis. They derived an expression for the modal loss factor from purely elastic analysis by suppressing the imaginary part of the complex stiffness. The proposed method takes the advantage of the relative ease of use and the ability of handling complex problems, compared to analytical solutions that are limited to simple geometries. Moreover, it is very useful for large-scale problems because of less computation time needed to solve real eigenvalue problems. The MSE method has some divergence in the results obtained for high values of the visco-elastic loss factor, but still in the acceptable range for engineering applications. Chen and Chan developed four types of composite finite elements to estimate the vibration and damping characteristics of beams, plates, and shells. In the same category, Rikards [10] developed a finite element model for Timoshenko beams and Mindlin-Reissner plates with constrained visco-elastic layers. It was also concluded that for a small cover ratio, separation of the visco-elastic layer for a center-covered beam generates a higher system loss factor than that without separation. Chen [11] adopted the topographical method in conjunction with the complex method to search for the optimal placement of constrained-layer damping (CLD) treatment for vibration suppression of plates. An objective function including structural damping ratios, resonant frequencies' shift, and CLD thickness is defined. Zheng [12] demonstrated an optimization study of constrained layer damping beams to minimize vibration energy (VE). The optimization method chosen was a "Genetic Algorithm" (GA) with penalty functions for the constraints. Zheng [13] presented a layout optimization of constrained layer damping (CLD) treatment for minimizing the vibration response of cylindrical shells. The optimization method chosen was still "Genetic Algorithm" (GA) with penalty functions. Although some researchers have addressed their studies on optimization of constrained layer damping treatments, the researches on this topic are still not enough and quite a few. The optimization of CLD treatment has been limited to simple sizing or shape optimization. In order to enhance the energy dissipation mechanism and improve the damping characteristics of vibrating structures, it is very significant to extend simple and local sizing or shape optimization to effective and global topological optimization.

Topology optimization has been a very active area of research since Bendsoe and Kikuchi [14] introduced the homogenization method. A complete literature review can be found in [15]. The number of solution methods were developed such as Optimality Criteria (OC) methods [16], Sequential Linear Programming (SLP) methods [17] or Method of Moving Asymptotes (MMA) and some others. Svanberg [18] introduced Method of Moving Asymptotes 

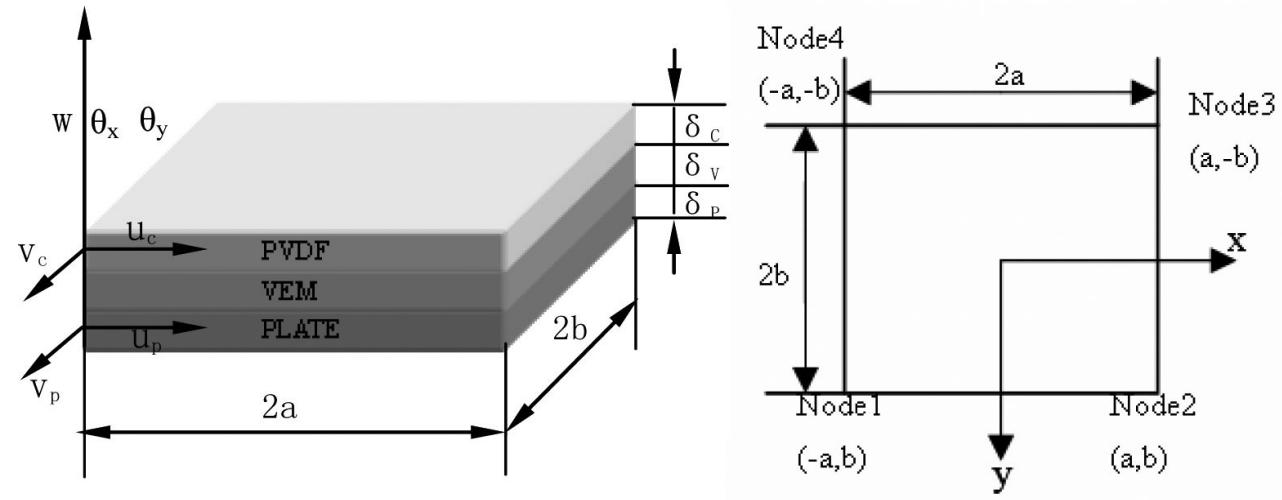

Fig. 1. A schematic drawing of an element in FE structure of plate with CLD treatments.

in 1987 and developed its new globally convergent version [19] in 1999. MMA is a mathematical programming algorithm and has proven itself to be versatile and well suited for large scale topology optimization problems. Bendsøe and Sigmund [20] developed Solid Isotropic Material with Penalization (SIMP) method which is proven to be very popular and extremely efficient interpolation scheme in order to obtain a clear distribution of material through a penalization power. In this model, the topology optimization problem is converted dramatically into a sizing problem on a fixed domain. Yang Deqing [21] proposed the concept of damping cell element, sensitivity analysis and Optimality Criteria (OC) method was used to determine the optimal topology of damping material on the plates. Mohammed [22] dealt with the optimal problem on constrained layer damping beams or plates using Inverse Homogenization Method (IHM).

The attempt of this paper is made to present a topology optimization approach to solve the optimal topology of constrained layer damping on plates to enhance the energy dissipation of vibrating structures. The governing equation of motion of constrained layer damping plates is derived on the basis of energy approach. A finite element model is developed and used along with an optimization algorithm in order to determine the optimal topologies of damping material layout on plates. In topology optimization formula, the damping material density and volume fraction are considered as design variable and constraint respectively. Meantime, the modal damping ratio of plates is assigned as the objective function. The sensitivity of modal damping ratio to design variable is further derived and Method of Moving Asymptote (MMA) is employed to search the optimal topologies of constrained layer damping on plates.

\section{Finite element modeling}

\subsection{The model}

Figure 1 is a schematic drawing of an element in finite element model of constrained layer damping plate. The geometry and deformation of the plate with CLD treatment is as shown in Fig. 2. The finite element model is developed based on the following assumption which were initially adopted by Mead and Markus [5] in deriving the governing equation of bending vibration of sandwich beams, and are common used for most researches of PCLD and ACLD treatments for structural vibration suppression.

(a) Shear strains in the constrained layer and the base layer are negligible, the constrained layer and the base plate are elastic and dissipate no energy.

(b) The visco-elastic layer undergoes transverse shear only and no normal stress. A linear and visco-elastic, frequency-independent, complex constant shear modulus

$$
G=G^{\prime}(1+\eta i)
$$

where $G^{\prime}, \eta$ and $i$ are the storage modulus, the loss factor and $\sqrt{-1}$ respectively, is used to describe the properties of the visco-elastic core layer. 


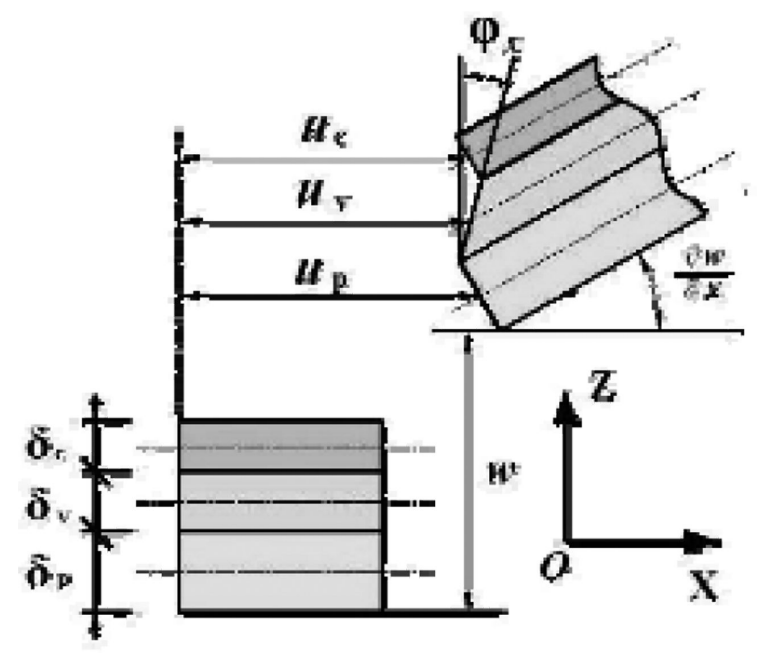

Fig. 2. Geometry and deformation of the plate with CLD treatment.

(c) All displacements are small compared to the structural dimensions thus linear theories of elasticity, viscoelasticity are adopted.

(d) All layers are considered to be perfectly bonded together, no slipping takes place at the interfaces.

(e) The plane transverse to the middle plane remains plane when bending.

(f) The transverse displacements of all points on any cross section of the sandwiched plate are considered to be equal.

\subsection{Strain-displacement relations}

The strain-displacement relations between layers, extended from the model of Mead and Markus [5], were derived to be:

$$
\begin{aligned}
& \varepsilon_{x x}^{i}=\frac{\partial u_{i}}{\partial x}-z \frac{\partial^{2} w}{\partial x^{2}} \\
& \varepsilon_{y y}^{i}=\frac{\partial v_{i}}{\partial y}-z \frac{\partial^{2} w}{\partial y^{2}} \\
& \varepsilon_{x y}^{i}=\frac{1}{2}\left(\frac{\partial u_{i}}{\partial y}+\frac{\partial v_{i}}{\partial x}\right)-z \frac{\partial^{2} w}{\partial x \partial y} \quad i=p, c \\
& \varepsilon_{z x}^{i}=\frac{d}{\delta_{v}} \frac{\partial w}{\partial x}+\frac{u_{c}-u_{p}}{\delta_{v}} \\
& \varepsilon_{z y}^{i}=\frac{d}{\delta_{v}} \frac{\partial w}{\partial y}+\frac{v_{c}-v_{p}}{\delta_{v}}
\end{aligned}
$$

where $d=\delta_{v}+\frac{1}{2}\left(\delta_{c}+\delta_{p}\right)$ is the distance from the plate's neutral surface to the CL's central surface.

\subsection{Shape functions}

The plate/CLD elements here are two-dimensional elements bounded by four nodal points. Each node has seven degrees of freedom to describe the longitudinal displacements of the constrained layer, $u_{c}, v_{c}$, the longitudinal displacements of the base plate, $u_{p}, v_{p}$, the transverse deflection $w$ and the slopes of the deflection line, $\theta_{x}, \theta_{y}$. The 
spatial distributions of the longitudinal displacements $u_{c}, v_{c}, u_{p}, v_{p}$ as well as the transverse deflection $w$ over any element $i$ of the plate with CLD treatment can be given by [23]

$$
\begin{aligned}
& u_{c}=a_{1}+a_{2} x+a_{3} y+a_{4} x y \\
& v_{c}=a_{5}+a_{6} x+a_{7} y+a_{8} x y \\
& u_{p}=a_{9}+a_{10} x+a_{11} y+a_{12} x y \\
& v_{p}=a_{13}+a_{14} x+a_{15} y+a_{16} x y \\
& w=a_{17}+a_{18} x+a_{19} y+a_{20} x^{2}+a_{21} x y+a_{22} y^{2}+a_{23} x^{3} \\
& +a_{24} x^{2} y+a_{25} x y^{2}+a_{26} y^{3}+a_{27} x^{3} y+a_{28} x y^{3} \\
& \theta_{x}=\frac{\partial w}{\partial y}, \theta_{y}=-\frac{\partial w}{\partial x}
\end{aligned}
$$

where the constants $\{a\}=\left\{\begin{array}{llll}a_{1} & a_{2} & \ldots & a_{28}\end{array}\right\}$ are determined in terms of the twenty eight components of the node deflection vector $\left\{\Delta^{e}\right\}$ of the ith element which is bonded by the nodes $1,2,3,4$ as shown in Fig. 1 . The node deflection vector $\left\{\Delta^{e}\right\}$ is given by

$$
\left\{\Delta^{e}\right\}=\left\{\Delta_{1} \Delta_{2} \Delta_{3} \Delta_{4}\right\}^{T}
$$

where

$$
\left\{\Delta_{i}\right\}=\left\{u_{c \mathbf{i}} v_{c i} u_{p i} v_{p i} w_{i} \theta_{x i} \theta_{y i}\right\}^{T} \text { for } i=1,2, \cdots, 4
$$

Therefore, the deflection $\{\Delta\}=\left\{u_{c} v_{c} u_{p} v_{p} w \theta_{x} \theta_{y}\right\}^{T}$ at any location $\{x, y\}$ inside the $i$ th element can be obtained from:

$$
\begin{aligned}
& \left\{u_{c} v_{c} u_{p} v_{p} w \theta_{x} \theta_{y}\right\}^{T} \\
& =\left\{\left[N_{1}\right]\left[N_{2}\right]\left[N_{3}\right]\left[N_{4}\right]\left[N_{5}\right]\left[N_{5}\right]_{, y}-\left[N_{5}\right]_{, x}\right\}^{T}
\end{aligned}
$$

where $\left[N_{1}\right],\left[N_{2}\right],\left[N_{3}\right],\left[N_{4}\right],\left[N_{5}\right],\left[N_{5}\right]_{, y}$ and $-\left[N_{5}\right]_{, x}$ are the spatial interpolating vectors corresponding to $u_{c}, v_{c}$, $u_{p}, v_{p}, w, \theta_{x}$ and $\theta_{y}$ respectively.

\subsection{Equation of motion}

The kinetic energies and potential energies of three layers can be expressed as follows:

Potential energies:

(a) base plate:

$$
T_{p}^{e}=\frac{1}{2} \rho_{p} \delta_{p} \iiint_{V}\left[\left(\frac{\partial u_{p}}{\partial t}\right)^{2}+\left(\frac{\partial v_{p}}{\partial t}\right)^{2}+\left(\frac{\partial w}{\partial t}\right)^{2}\right] \mathrm{d} V=\frac{1}{2}\left\{\dot{\Delta}^{(e)}\right\}^{T}\left[M_{p}^{(e)}\right]\left\{\dot{\Delta}^{(e)}\right\}
$$

(b) constrained layer:

$$
T_{c}^{e}=\frac{1}{2} \rho_{c} \delta_{c} \iiint_{V}\left[\left(\frac{\partial u_{c}}{\partial t}\right)^{2}+\left(\frac{\partial v_{c}}{\partial t}\right)^{2}+\left(\frac{\partial w}{\partial t}\right)^{2}\right] \mathrm{d} V=\frac{1}{2}\left\{\dot{\Delta}^{(e)}\right\}^{T}\left[M_{c}^{(e)}\right]\left\{\dot{\Delta}^{(e)}\right\}
$$

(c) viscoelastic layer:

$$
T_{v}^{e}=\frac{1}{2} \rho_{v} \delta_{v} \iiint_{V}\left[\left(\frac{\partial u_{v}}{\partial t}\right)^{2}+\left(\frac{\partial v_{v}}{\partial t}\right)^{2}+\left(\frac{\partial w}{\partial t}\right)^{2}\right] \mathrm{d} V=\frac{1}{2}\left\{\dot{\Delta}^{(e)}\right\}^{T}\left[M_{v}^{(e)}\right]\left\{\dot{\Delta}^{(e)}\right\}
$$

Kinetic energies: 
(a) base plate:

$$
E_{p}^{e}=\frac{1}{2} \iiint_{V} \varepsilon_{p}^{* \mathrm{~T}} \sigma_{p} \mathrm{~d} V=\frac{1}{2}\left\{\Delta^{(e)}\right\}^{T}\left[K_{p}^{(e)}\right]\left\{\Delta^{(e)}\right\}
$$

(b) constrained layer:

$$
E_{c}^{e}=\frac{1}{2} \iiint_{V} \varepsilon_{c}^{* \mathrm{~T}} \sigma_{c} \mathrm{~d} V=\frac{1}{2}\left\{\Delta^{(e)}\right\}^{T}\left[K_{c}^{(e)}\right]\left\{\Delta^{(e)}\right\}
$$

(c) visco-elastic layer:

$$
\begin{aligned}
& E_{v}^{e}=\frac{1}{2} \iiint_{V} \varepsilon_{v}^{* \mathrm{~T}} \sigma_{v} \mathrm{~d} V=\frac{1}{2}\left\{\Delta^{(e)}\right\}^{T}\left[K_{v}^{(e)}\right]\left\{\Delta^{(e)}\right\} \\
& E_{\beta v}^{e}=\frac{1}{2} \iiint_{V}\left(G \beta_{x}^{2}+G \beta_{y}^{2}\right) \mathrm{d} V=\frac{1}{2}\left\{\Delta^{(e)}\right\}^{T}\left[K_{\beta v}^{(e)}\right]\left\{\Delta^{(e)}\right\}
\end{aligned}
$$

The work done by external disturbance force in the plate/CLD element is expressed:

$$
W_{f}^{e}=\left\{\Delta^{(e)}\right\}^{T} f^{(e)}
$$

where $f^{(e)}$ is external disturbance force vector of the plate/CLD element.

The governing equations of motion for the plate/CLD element can be derived by applying Lagrange's Equations [24]. The result is given by:

$$
\left[M^{(e)}\right]\left\{\ddot{\Delta}^{(e)}\right\}+\left[K^{(e)}\right]\left\{\Delta^{(e)}\right\}=\left\{f^{(e)}\right\}
$$

where $\left[M^{(e)}\right]$ and $\left[K^{(e)}\right]$ denote mass and stiffness matrices of the plate/CLD element. These matrices can be written by:

$$
\begin{aligned}
& {\left[M^{(e)}\right]=\left[M_{p}^{(e)}\right]+\left[M_{v}^{(e)}\right]+\left[M_{c}^{(e)}\right]} \\
& {\left[K^{(e)}\right]=\left[K_{p}^{(e)}\right]+\left[K_{v}^{(e)}\right]+\left[K_{\beta v}^{(e)}\right]+\left[K_{c}^{(e)}\right]}
\end{aligned}
$$

Assembly of mass and stiffness matrices yields global mass and stiffness matrices. The global mass and stiffness matrices as well as equation of motion for entire plate/CLD system are given:

$$
\begin{aligned}
& K=\sum_{e=1}^{n}\left(K_{p}^{(e)}+K_{v}^{(e)}+K_{\beta v}^{(e)}+K_{c}^{(e)}\right) \\
& M=\sum_{e=1}^{n}\left(M_{p}^{(e)}+M_{v}^{(e)}+M_{c}^{(e)}\right) \\
& {[M]\{\ddot{\Delta}\}+[K]\{\Delta\}=\{F\}}
\end{aligned}
$$

where $[K]$ and $[M]$ are the global mass and stiffness matrices respectively. Also, $\{F\}$ is external disturbance force vector of the plate/CLD system. 


\section{Topology optimization of constrained layer damping on plates}

The main objective in vibration damping is to dissipate the vibrational energy, which can be achieved by maximizing the modal damping ratio or minimizing the amplitude of the frequency response [22]. In terms of the modal damping ratio, an objective function can be established by maximizing the modal damping ratio of the structure:

$$
f=\sum_{r=1}^{m} \xi_{r}
$$

where $f$ denotes the objective function of the optimization problem in present study. $\xi_{r}$ is the $r$-th modal damping ratio of the damping structure. $m$ represents the number of the considered modal damping ratio. Define the relative density of each VEM element as the design variable vector:

$$
\rho=\left\{\rho_{1}, \rho_{2}, \cdots, \rho_{N}\right\}^{T}
$$

In addition, the constraint should be considered to limit the consumption of VEM, the volume fraction is limited here. Therefore, the optimization problem is formulated as follows:

$$
\begin{aligned}
& \text { find: } \rho=\left\{\rho_{1}, \rho_{2}, \ldots, \rho_{N}\right\}^{T} \in R \\
& \text { max }: f=\sum_{r=1}^{m} \xi_{r} \\
& \text { s.t. }:\left\{\begin{array}{l}
\sum_{i=1}^{n} \rho_{e}-V_{0} \alpha \leq 0 \\
\left(K-\omega_{j}^{2} M\right) \Phi_{j}=0 \\
0 \leqslant \rho_{e} \leqslant 1, e=1,2, \ldots, N
\end{array}\right.
\end{aligned}
$$

where $N$ is the number of elements, $\Phi_{j}$ is the eigenvector, $M$ and $K$ are the global mass and stiffness matrices, $V / V_{0}=\alpha$ is the volume fraction of visco-elastic material on the plate.

According to Solid Isotropic Material with Penalization (SIMP) method [25,26], the element mass and stiffness matrices can be expressed as the product of variable density and the entity element mass and stiffness matrices. The penalty factors are $p, q ; p, q \geqslant 1$. These penalty factors are used to accelerate the convergence of iteration results and obtain a clear pattern of the damping material on the plate.

$$
M_{v}\left(\rho_{e}\right)=\rho_{e}^{p} M_{v}^{(e)}, K_{v}\left(\rho_{e}\right)=\rho_{e}^{q} K_{v}^{(e)}
$$

where $\rho_{e}$ is variable density of each VEM element, it is a relative quantity and $0 \leqslant \rho_{e} \leqslant 1 . \rho_{e}=0$ means no VEM in this element or the thickness of VEM is equal to zero. $\rho_{e}=1$ means that the thickness of VEM in this element is equal to the assigned thickness. $M_{v}^{(e)}, K_{v}^{(e)}$ are the mass and stiffness matrices of VEM element. Keeping the base plate and constrained layer unchanged, the global mass and stiffness matrices can be calculated as follows:

$$
\begin{aligned}
& M=\sum_{e=1}^{n}\left(M_{p}^{(e)}+\rho_{e}^{p} M_{v}^{(e)}+M_{c}^{(e)}\right) \\
& K=\sum_{e=1}^{n}\left(K_{p}^{(e)}+\rho_{e}^{q}\left(K_{v}^{(e)}+K_{\beta v}^{(e)}\right)+K_{c}^{(e)}\right)
\end{aligned}
$$

where $p$ and $q$ are penalty factors, $p$ is $1, q$ is 3 . The layout of VEM on the plate can be determined by searching the optimal relative density of each VEM element. In order to solve the presented optimization problem, Method of Moving Asymptote (MMA) is employed. 


\section{Sensitivity analysis}

According to modal strain energy method [9], we can get the approximate expressions of the $r$-th modal damping ratio. The equation of motion for plate/CLD system is given:

$$
M \ddot{X}+\left(K_{R}+j K_{I}\right) X=0
$$

where $K_{R}$ is the real part of global stiffness matrix, $K_{I}$ is the imaginary part of global stiffness matrix. Therefore, the $r$-th modal damping ratio can be written:

$$
\xi_{r}=\frac{1}{2} \frac{\Phi_{r}^{T} K_{I} \Phi_{r}}{\Phi_{r}^{T} K_{R} \Phi_{r}}
$$

where $\Phi_{r}$ is the $r$-th eigenvector. As $\Phi_{r}$ does not depend on the variable density $\rho_{i}$ very much, $\frac{\partial \Phi_{r}}{\partial \rho_{i}}$ is assumed to be zero. Therefore, the derivatives of the $r$-th modal damping ratio to design variable $\rho_{i}$ is derived:

$$
\frac{\partial\left(\xi_{r}\right)}{\partial \rho_{i}}=\frac{1}{2} \frac{\left(\Phi_{r}^{T} \frac{\partial K_{I}}{\partial \rho_{i}} \Phi_{r}\right)\left(\Phi_{r}^{T} K_{R} \Phi_{r}\right)-\left(\Phi_{r}^{T} K_{I} \Phi_{r}\right)\left(\Phi_{r}^{T} \frac{\partial K_{R}}{\partial \rho_{i}} \Phi_{r}\right)}{\left(\Phi_{r}^{T} K_{R} \Phi_{r}\right)^{2}}
$$

The derivatives of stiffness matrix are obtained by solving the following sensitivity equations:

$$
\begin{aligned}
& \frac{\partial K_{I}}{\partial \rho_{i}}=\operatorname{Im} \sum_{i=1}^{n} q \rho_{i}^{(q-1)} K v \\
& \frac{\partial K_{R}}{\partial \rho_{i}}=R e \sum_{i=1}^{n} q \rho_{i}^{(q-1)} K v
\end{aligned}
$$

when $p=1, q=3$, the derivative of modal damping ratio can be expressed as follows:

$$
\frac{\partial\left(\xi_{r}\right)}{\partial \rho_{i}}=\frac{1}{2} \frac{\left(\Phi_{r}^{T} \operatorname{Im}\left(3 \rho_{i}^{2} K v\right) \Phi_{r}\right)\left(\Phi_{r}^{T} \operatorname{Re}\left(\rho_{i}^{3} K v+K p+K c\right) \Phi_{r}\right)-\left(\Phi_{r}^{T} \operatorname{Im}\left(\rho_{i}^{3} K v\right) \Phi_{r}\right)\left(\Phi_{r}^{T} \operatorname{Re}\left(3 \rho_{i}^{2} K v\right) \Phi_{r}\right)}{\left(\Phi_{r}^{T} \operatorname{Re}\left(\rho_{i}^{3} K v+K p+K c\right) \Phi_{r}\right)^{2}}
$$

The dynamic constraint function is given in the form:

$$
f_{j}=\Phi_{j}^{T}\left(K-\omega_{j}^{2} M\right) \Phi_{j}
$$

Furthermore, the derivative of the dynamic constraint function is calculated as follows:

$$
\begin{aligned}
& \frac{\partial f_{j}}{\partial \rho_{i}}=\Phi_{j}^{T}\left(\frac{\partial K}{\partial \rho_{i}}-\omega_{j}^{2} \frac{\partial M}{\partial \rho_{i}}\right) \Phi_{j} \\
& \frac{\partial M}{\partial \rho_{i}}=\sum_{i=1}^{n} p \rho_{i}^{(p-1)} M v
\end{aligned}
$$

when $p=1, q=3$, the sensitivity of the constrained functions is yielded:

$$
\frac{\partial f_{j}}{\partial \rho_{i}}=\Phi_{j}^{T}\left(\sum_{i=1}^{n} 3 \rho_{i}^{2} K v-\omega_{j}^{2} \sum_{i=1}^{n} M v\right) \Phi_{j}
$$

\section{Numerical examples}

The model developed in the previous sections is applied to some cases. The adopted optimization procedures are summarized in block diagram at Fig. 3. The procedures rely on using the Method of Moving Asymptotes (MMA) proposed by Svanberg $[18,19]$ to determine the optimal distribution of damping material on the plate. It is noted that the computation time is mainly determined by building/solving the finite element model with the complex stiffness matrices and using the MMA approach. Some numerical methods and techniques such as numerical differential used in the procedure can be applied to short the computation time when the total number of elements becomes large. 
Table 1

Physical and geometrical parameters of plate/ CLD system

\begin{tabular}{lccccc}
\hline layer & Length $(\mathrm{m})$ & Width $(\mathrm{m})$ & Thickness $(\mathrm{mm})$ & Density $\left(\mathrm{kg} / \mathrm{m}^{3}\right)$ & Modulus $(\mathrm{MPa})$ \\
\hline base plate & 0.25 & 0.125 & 0.5 & 2700 & $7100^{\mathrm{b}}$ \\
Viscoelastic & 0.25 & 0.125 & 0.5 & 1140 & $20^{\mathrm{a}}$ \\
PVDF & 0.25 & 0.125 & 0.028 & 1800 & $2250^{\mathrm{b}}$ \\
\hline
\end{tabular}

${ }^{\mathrm{a}}$ shear modulus, $\eta=0.5$; ${ }^{\mathrm{b}}$ Young's modulus.

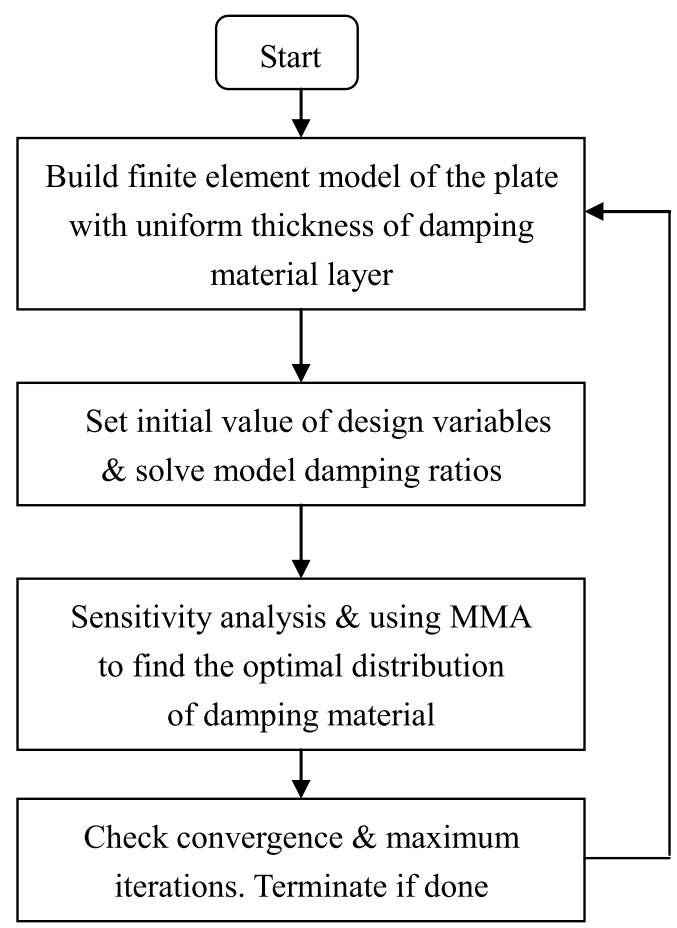

Fig. 3. Block diagram for the optimization procedures.

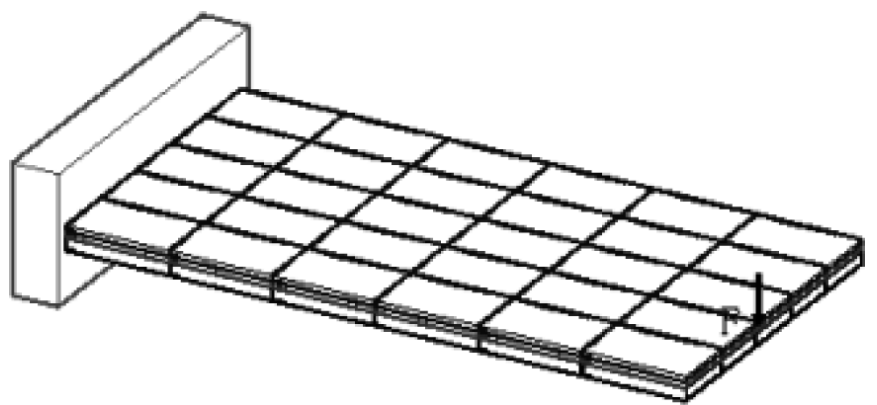

Fig. 4. A cantilever plate/CLD system.

\subsection{Cantilever plate/CLD system}

A cantilever plate/CLD system shown as Fig. 4 is considered as the first numerical example. Table 1 lists the main physical and geometrical parameters of the base plate, the visco-elastic layer and constrained layer. The plate is made of aluminum and is treated with visco-elastic sheets of DYAD606 from SOUNDCOAT Company. These visco-elastic sheets are constrained by a continuous active polymeric film from AMP, Inc (Valley Forge, PA, USA). 


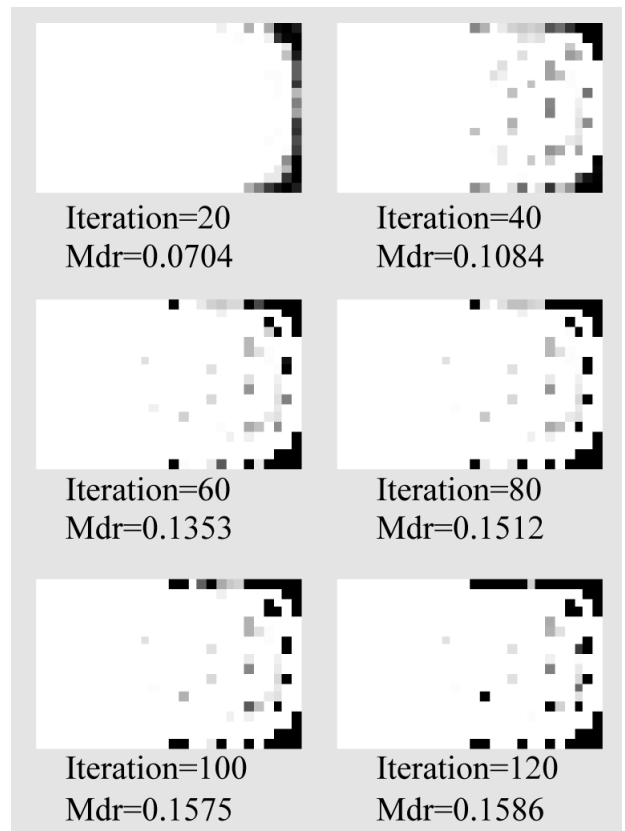

Fig. 5. Optimal distribution of damping material at different iteration numbers, $v f=0.2$.

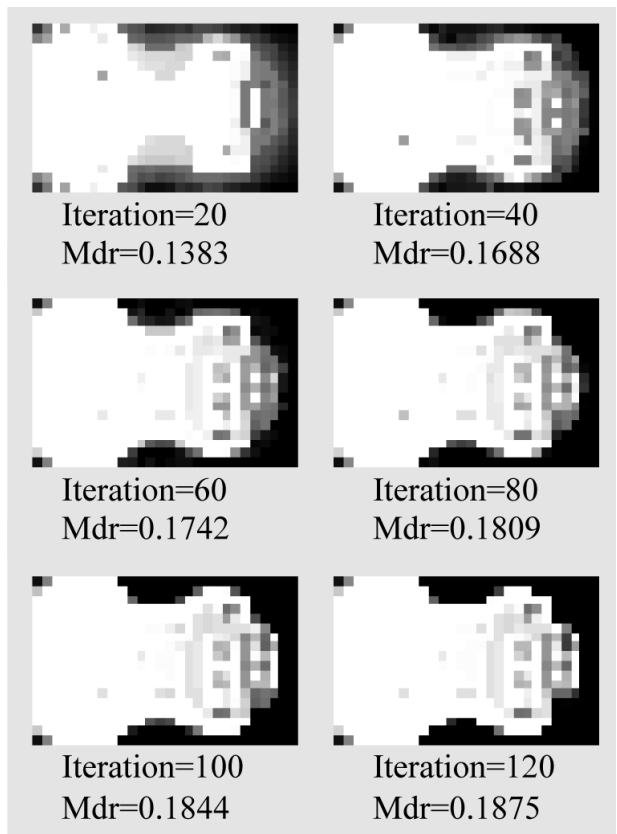

Fig. 6. Optimal distribution of damping material at different iteration numbers, $v f=0.4$.

In optimization process, the optimal topology of visco-elastic layer is solved by Method of Moving Asymptote (MMA) approach. The volume fraction of visco-elastic material is fixed as the constraint condition.

Figure 5 shows some of the intermediate and final iterations of visco-elastic material layout on the cantilever plate when the first modal damping ratio is maximized. It is clear that the free short edge to opposite fixed edge in the cantilever plate should be covered partially with visco-elastic material. No visco-elastic materials are needed in the 


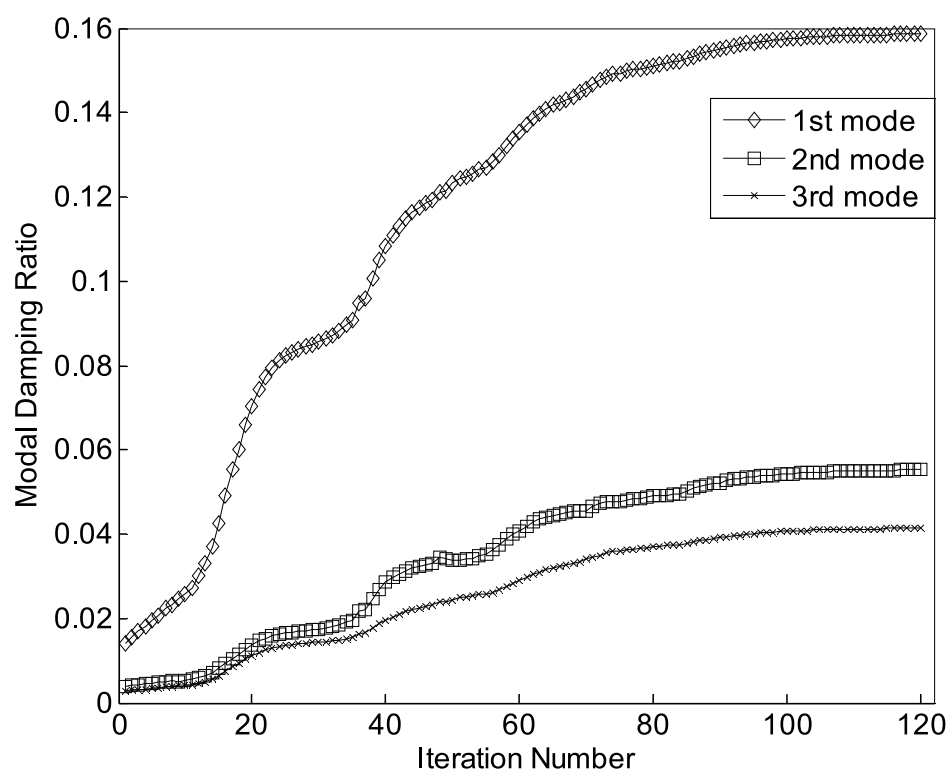

Fig. 7. Iteration histories for different modes $v f=0.2$.

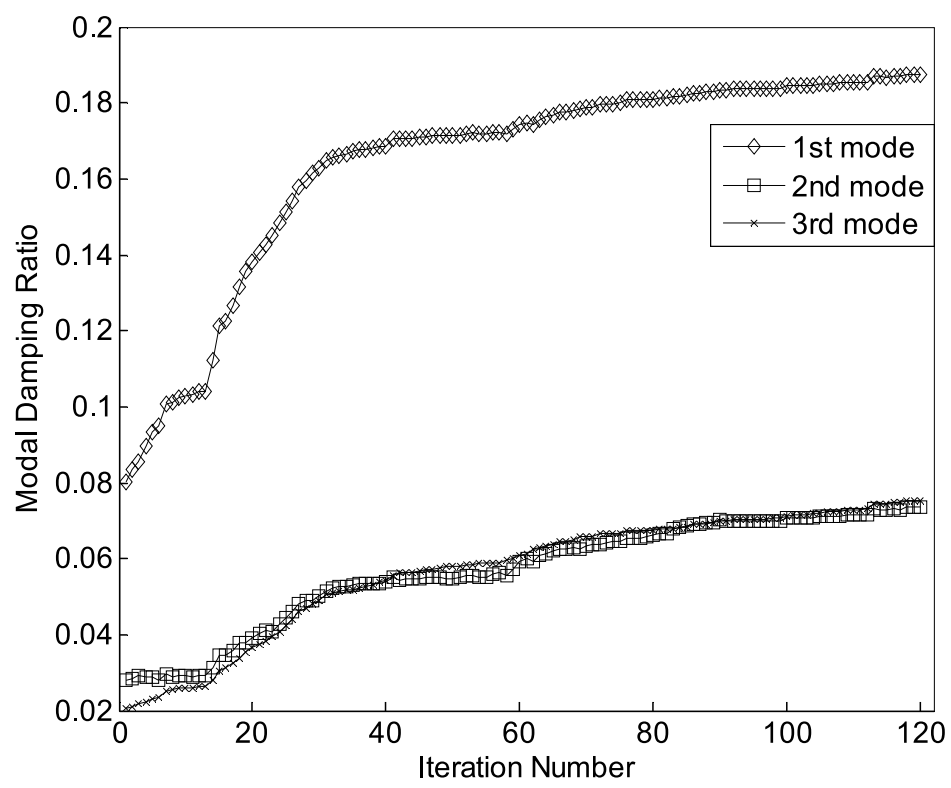

Fig. 8. Iteration histories for different modes $v f=0.4$.

center of the plate. In this case, volume fraction is fixed to 0.2. Figure 7 shows the evaluation of the first three modal damping ratios along the optimization process. It can be seen that these modal damping ratios increase with iteration numbers gradually until the maximums are achieved.

Figure 6 shows some of the intermediate and final iterations of visco-elastic material layout when the first modal damping ratio is maximized and volume fraction is changed to 0.4 . It can be seen that the optimal locations to cover visco-elastic material are extended from the short free edge to the opposite fixed edge along the two free long edges. Figure 8 reflects the evaluation process of the first three modal damping ratios when iteration numbers increase.

Figure 9 shows some of the intermediate and final iterations of visco-elastic material pattern to maximize the first modal damping ratio where volume fraction is increased to 0.6. It is clear that the optimal locations to cover 


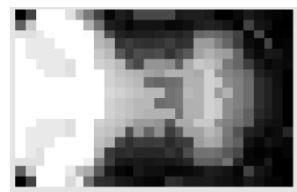

Iteration $=20$

$\mathrm{Mdr}=0.1715$

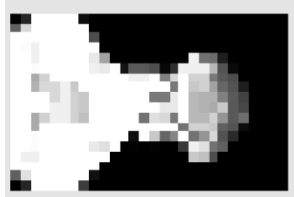

Iteration $=60$

$\mathrm{Mdr}=0.1966$

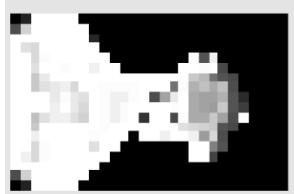

Iteration $=100$

$\mathrm{Mdr}=0.2038$

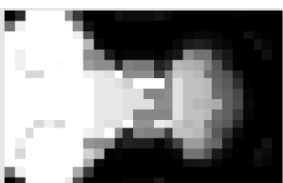

Iteration $=40$

$\mathrm{Mdr}=0.1865$

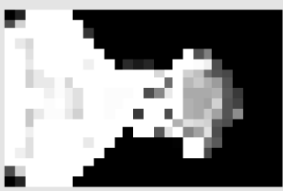

Iteration $=80$

$\mathrm{Mdr}=0.2024$

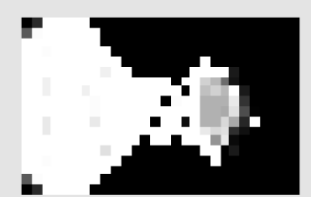

Iteration $=120$

$\mathrm{Mdr}=0.2054$

Fig. 9. Optimal distribution of damping material at different iteration numbers, $v f=0.6$.

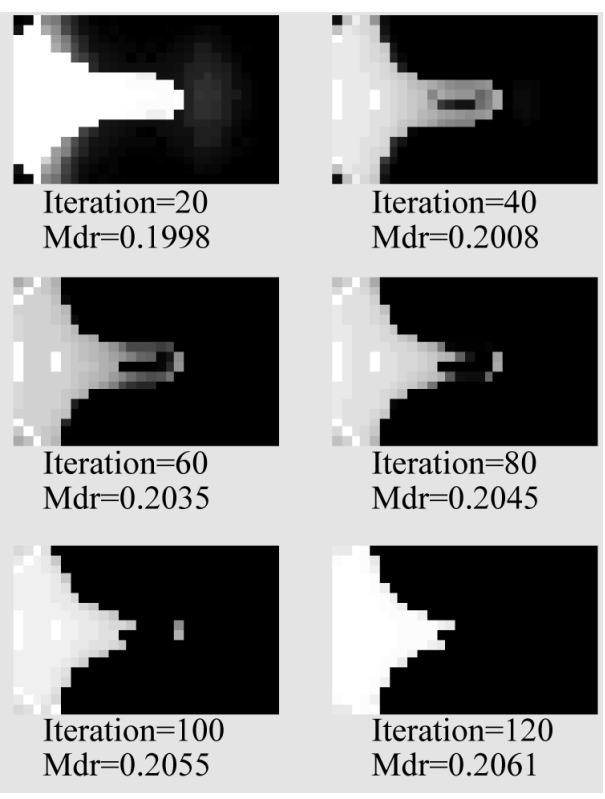

Fig. 10. Optimal distribution of damping material at different iteration numbers, $v f=0.8$.

visco-elastic material are extended more than that of the previous case with volume fraction 0.4 from the short free edge to the opposite fixed edge. The empty areas are concentrated in the center or the fixed edge of the plate. Figure 11 displays the iteration history behind the optimum solutions in Fig. 9. It is noted that when the volume fraction increases, the first three modal damping ratios increase at a stable rate. This implies that the volume fraction plays an important role to reach higher modal damping ratios of plate/CLD system when the same optimization procedures are applied.

Figure 10 shows some of the intermediate and final iterations of visco-elastic material pattern when volume 


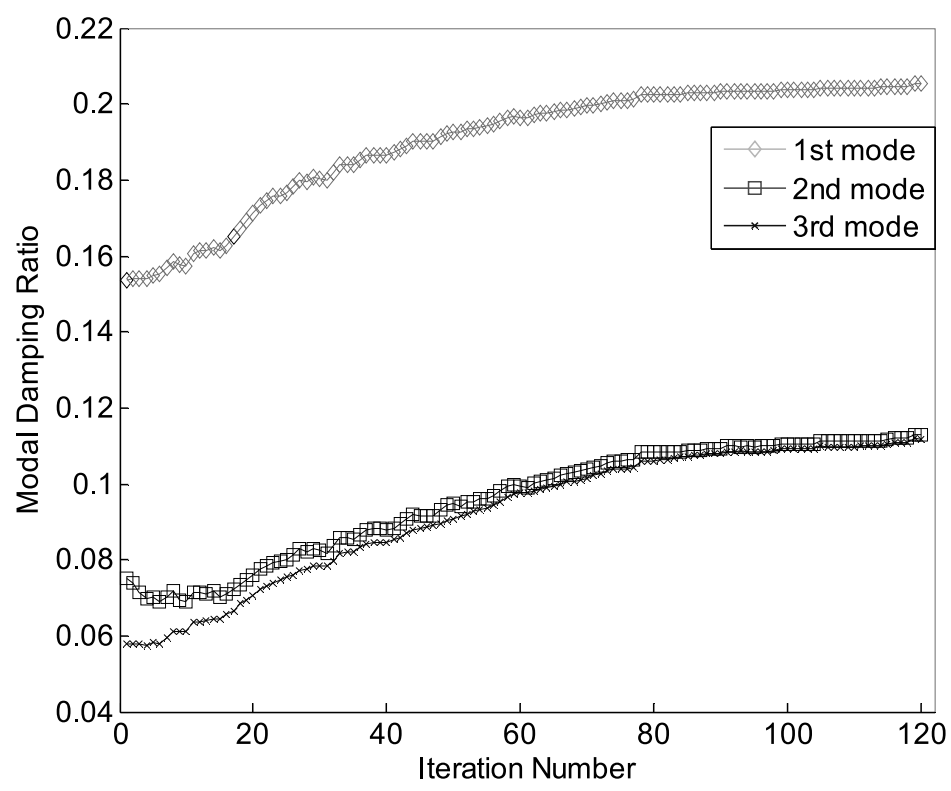

Fig. 11. Iteration histories for different modes $v f=0.6$.

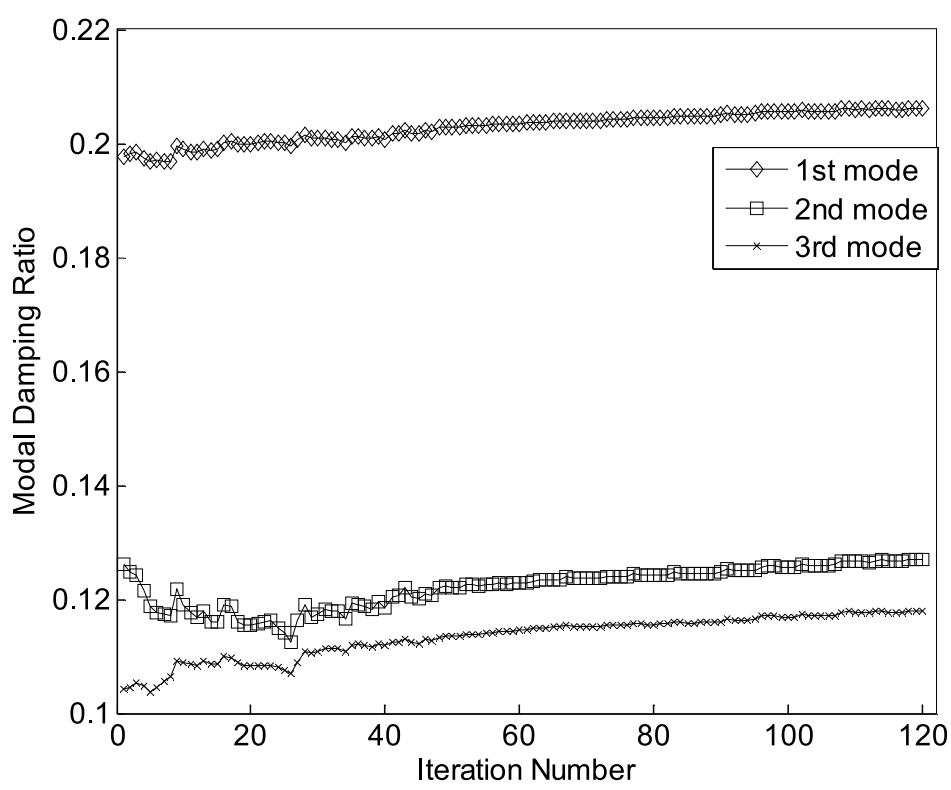

Fig. 12. Iteration histories for different modes $v f=0.8$.

fraction is achieved to 0.8 . It is obvious that the optimal locations to cover visco-elastic material have been extended to the fixed edge through the center of the plate. The optimal topology is still symmetric. Figure 12 shows the iteration history. The same phenomenon is observed. When the volume fraction is increased continuously, the modal damping ratios also increase. This means that the volume fraction is a key constraint which determines the optimal topology of damping material and available maximum value of modal damping ratios.

Figure 13 displays frequency response of the cantilever plate/CLD system at the mid-point of the free short edge excited by unit harmonic force at the same point. A detail and enlarged description can be seen from Figs 14 to 16. It is seen that the displacement of the mid-point of the free short edge decreases rapidly when the cantilever 


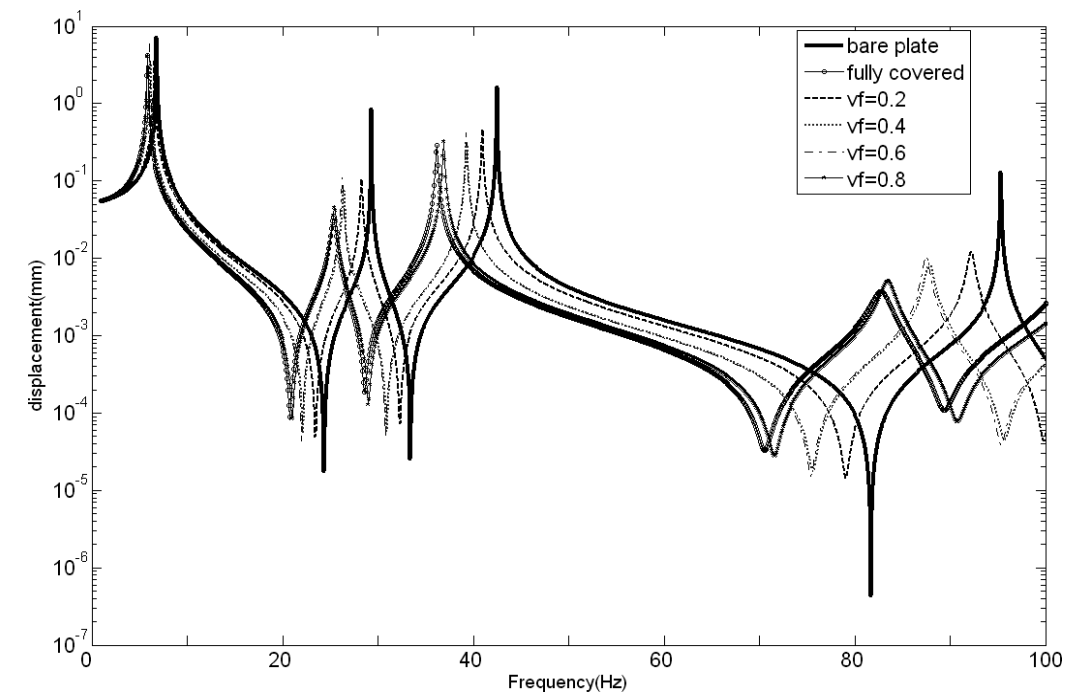

Fig. 13. Frequency response at the mid-point of free short edge excited by unit harmonic force at the same point.

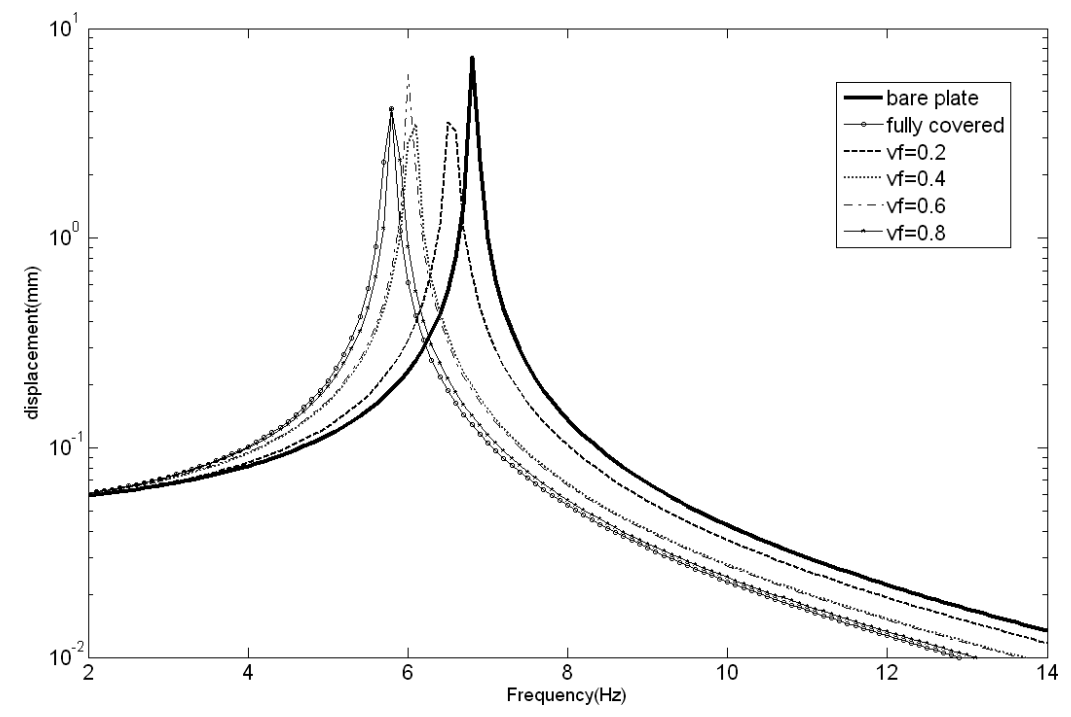

Fig. 14. The first natural frequency.

plate/CLD system is compared with the cantilever bare plate. However, once the volume fraction is reached to 0.4 , the displacement of observed point will not increase significantly. Specially, when the first or the third mode is focused on, the difference of the displacement is a little bit small. This means that the fully covered damping material on the plate is not necessary if the proposed optimization procedures are applied and the optimal pattern of damping material layout is determined. Therefore, the proposed method can be an invaluable tool to achieve a balance between low additional mass and reasonable vibration control action.

\subsection{Simple supported plate/CLD system}

Figure 17 is a configuration of simple supported plate/CLD system, it is considered as the second case to test the effectiveness of the proposed optimization approach. The same materials with cantilever plate/CLD system are applied here. 


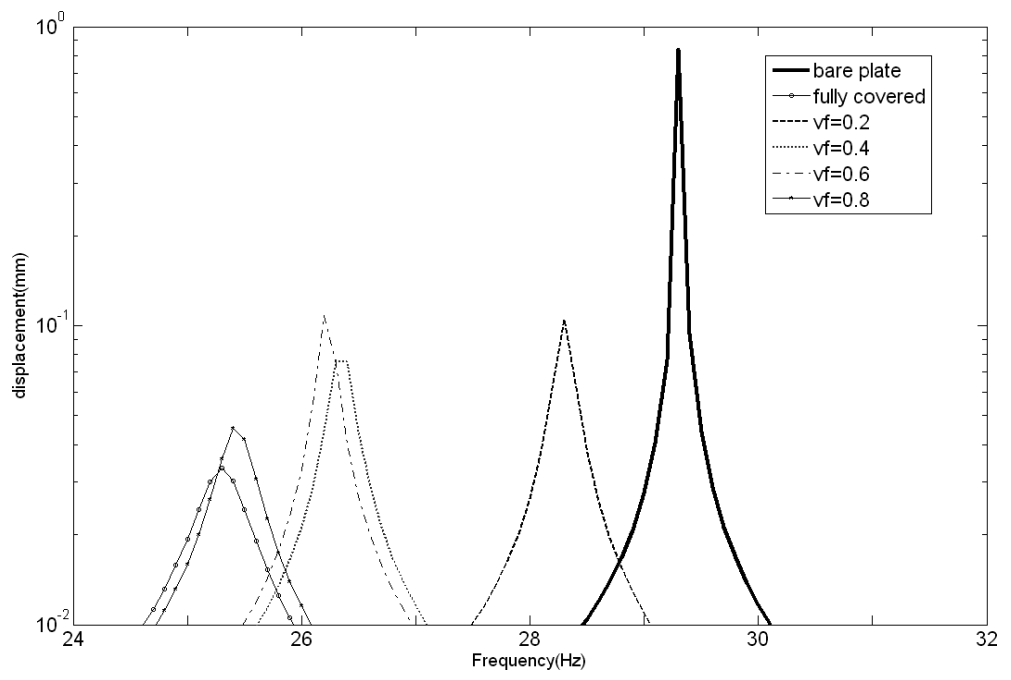

Fig. 15. The second natural frequency.

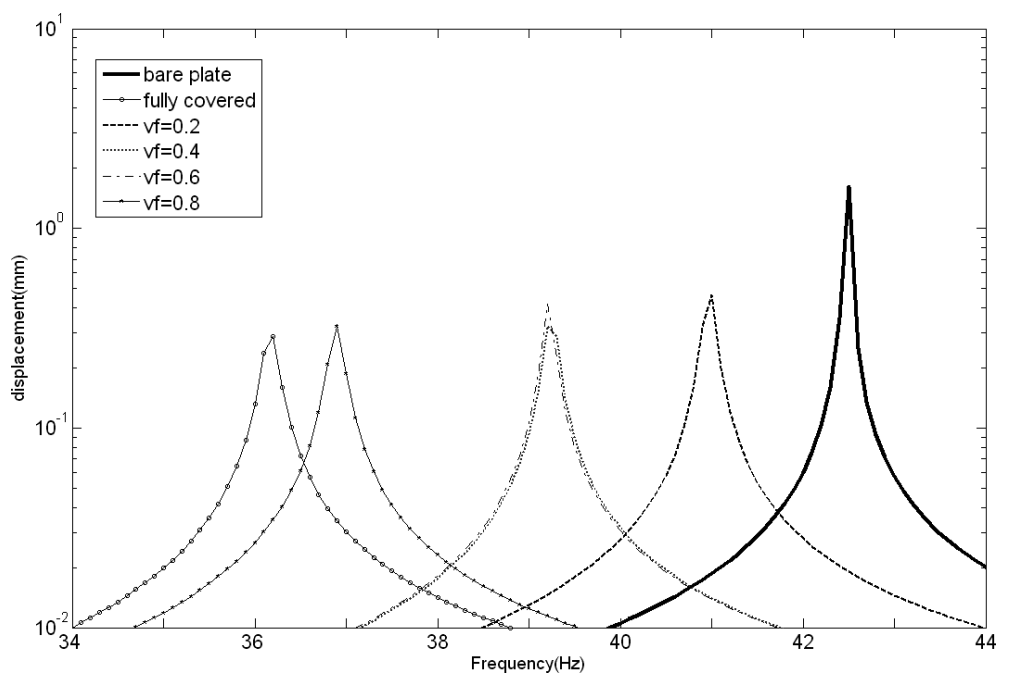

Fig. 16. The third natural frequency.

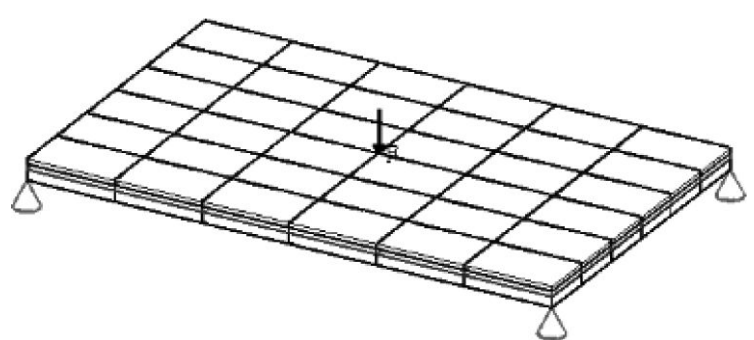

Fig. 17. Simply supported plate/CLD system.

Figure 18 displays the optimal pattern of damping material when the boundary condition of the plate is changed from one of the short edges clamped to four corners of the plate simple supported. It can be seen that when iteration number is increased, the optimal pattern becomes more clear than previous iterations until a final optimum solution 


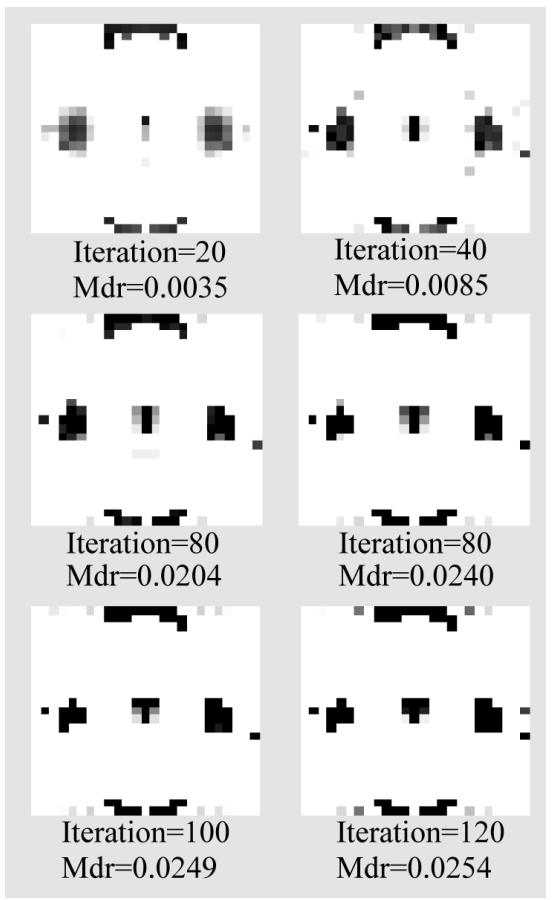

Fig. 18. Optimal distribution of damping material at different iteration numbers, $v f=0.2$.

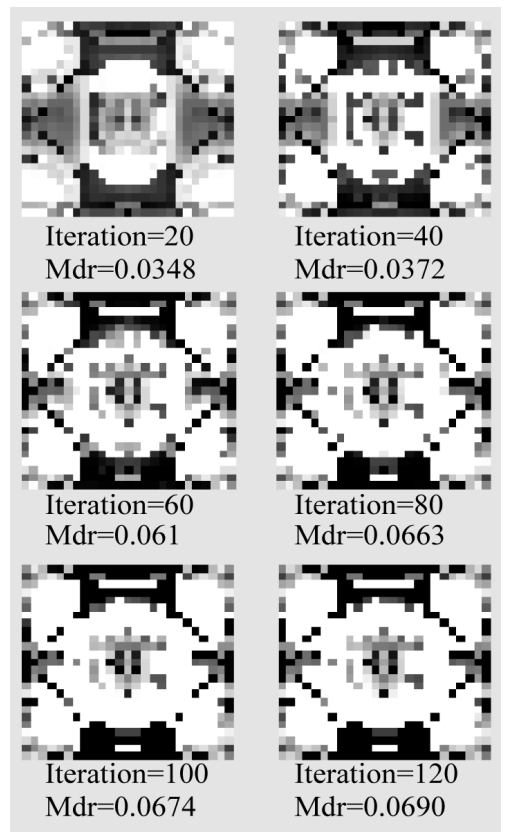

Fig. 19. Optimal distribution of damping material at different iteration numbers, $v f=0.4$.

is obtained. In this situation, the volume fraction is limited to 0.2 . This means that only $20 \%$ damping material is allowed to cover on the plate. Figure 20 shows the evaluation of the first three modal damping ratios along the optimization process.

Figure 19 displays some of the intermediate and final iterations of visco-elastic material layout on the simple 


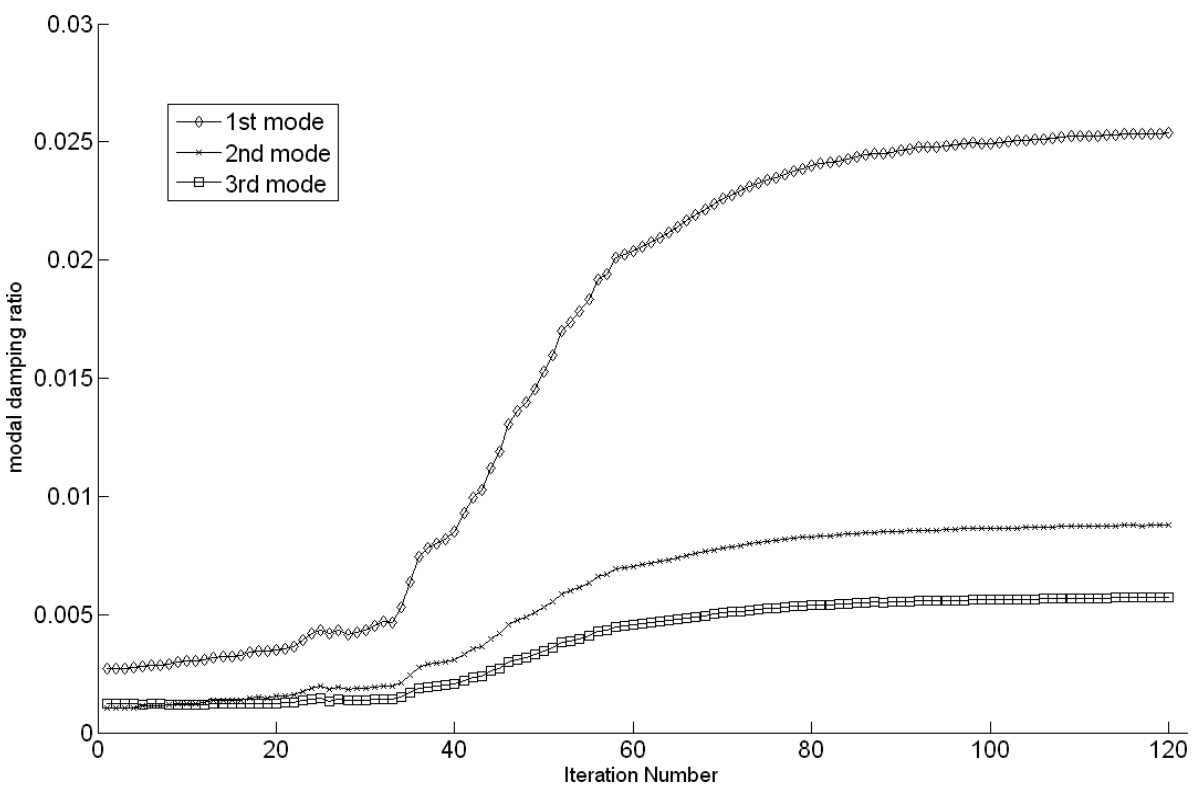

Fig. 20. Iteration histories for different modes $v f=0.2$.

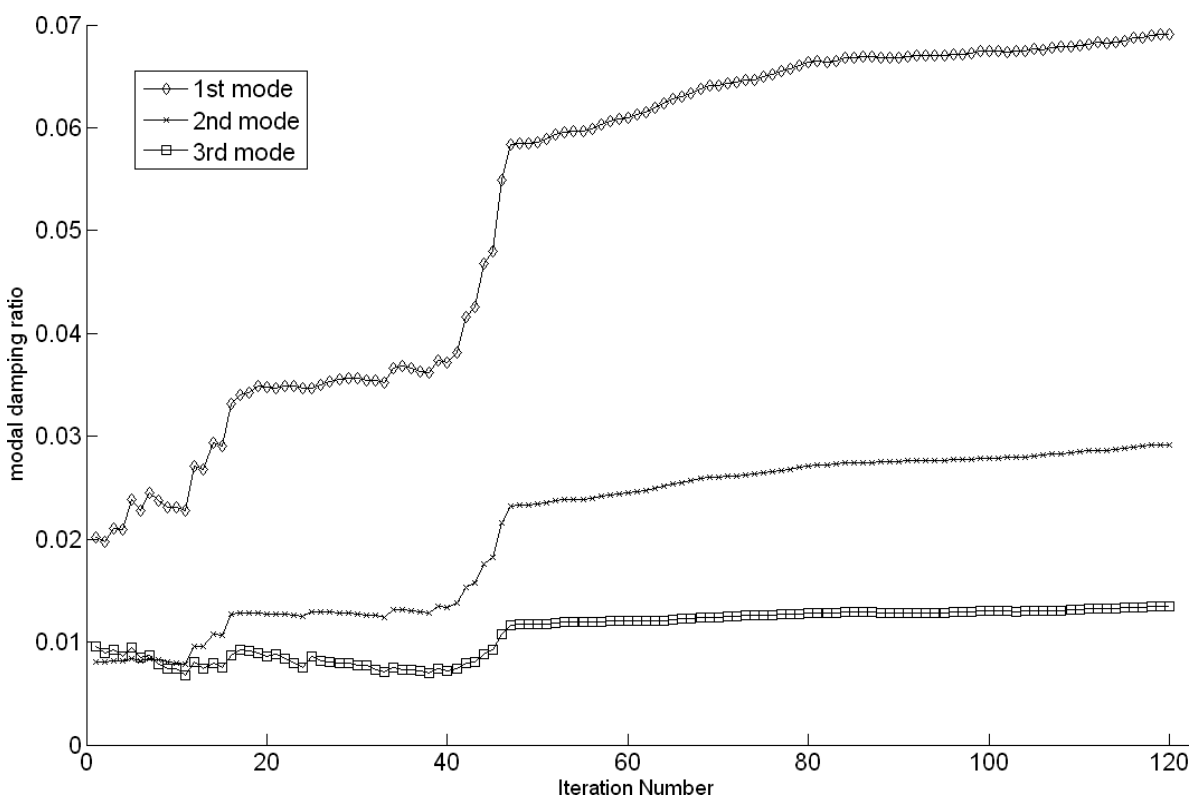

Fig. 21. Iteration histories for different modes $v f=0.4$.

supported plate when the first modal damping ratio is maximized. However, the black area increases compared to Fig. 18 due to a higher volume fraction of damping material is applied. Figure 21 demonstrates an evolved optimization process, a steady and dramatic rise of modal damping ratios can be observed.

Figures 22 and 23 display the intermediate and final optimal distributions of damping material on the plate when the volume fractions are limited to 0.6 and 0.8 respectively. It can be seen that the optimal positions of damping material are extended from the center of the plate to sides and corners of the plate due to the simple supported boundary condition in this case. Figures 24 and 25 demonstrate their iteration histories. The values of modal damping ratio for different modes increase dramatically with the volume fraction of damping material. 


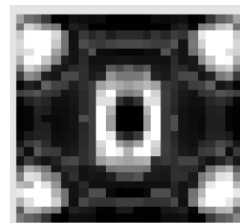

Iteration $=20$

$\mathrm{Mdr}=0.0592$

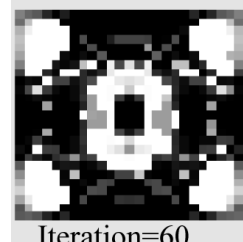

Iteration $=60$

$\mathrm{Mdr}=0.0814$

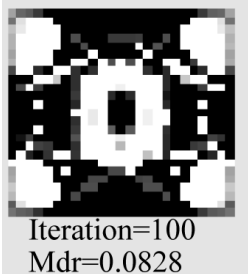

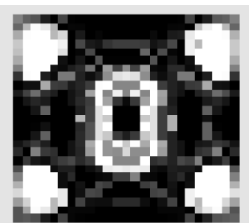

Iteration $=40$

$\mathrm{Mdr}=0.0665$

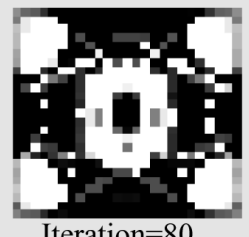

Iteration $=80$

$\mathrm{Mdr}=0.0814$

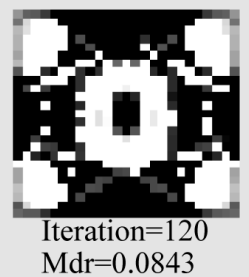

Fig. 22. Optimal distribution of damping material at different iteration numbers, $v f=0.6$.

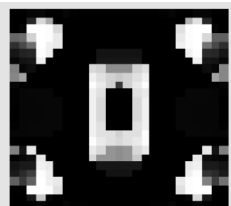

Iteration $=20$ $\mathrm{Mdr}=0.1038$

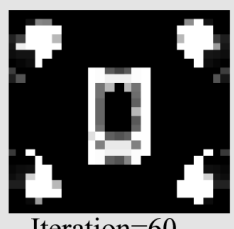

Iteration $=60$

$M d r=0.0982$

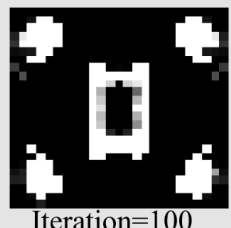

Iteration $=100$

$\mathrm{Mdr}=0.1155$

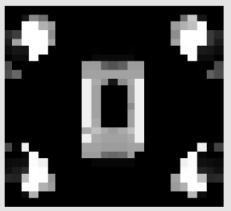

Iteration $=40$ $\mathrm{Mdr}=0.1053$

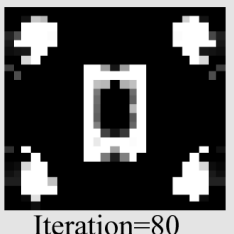

Iteration $=80$

$\mathrm{Mdr}=0.1108$

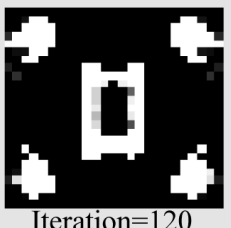

Iteration $=120$

$\mathrm{Mdr}=0.1157$

Fig. 23. Optimal distribution of damping material at different iteration numbers, $v f=0.8$.

Figure 26 displays frequency response of the simple supported plate/CLD system at the center of the plate excited by unit harmonic force at the same point. A detail and enlarged description can be seen from Figs 27 to 29 . It can be noted that the displacement at the center of the plate reduces rapidly when the plate is partially covered by damping material. It reflects the action of damping material in vibration energy dissipation. However, the difference 


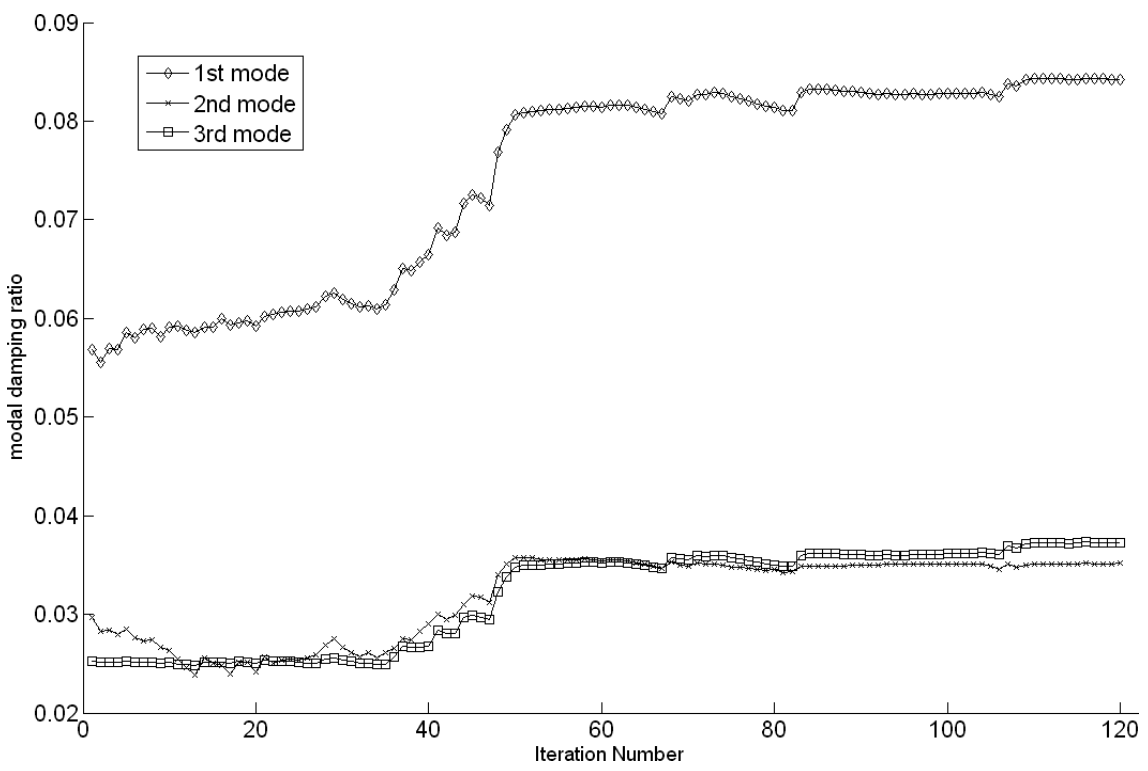

Fig. 24. Iteration histories for different modes $v f=0.6$.

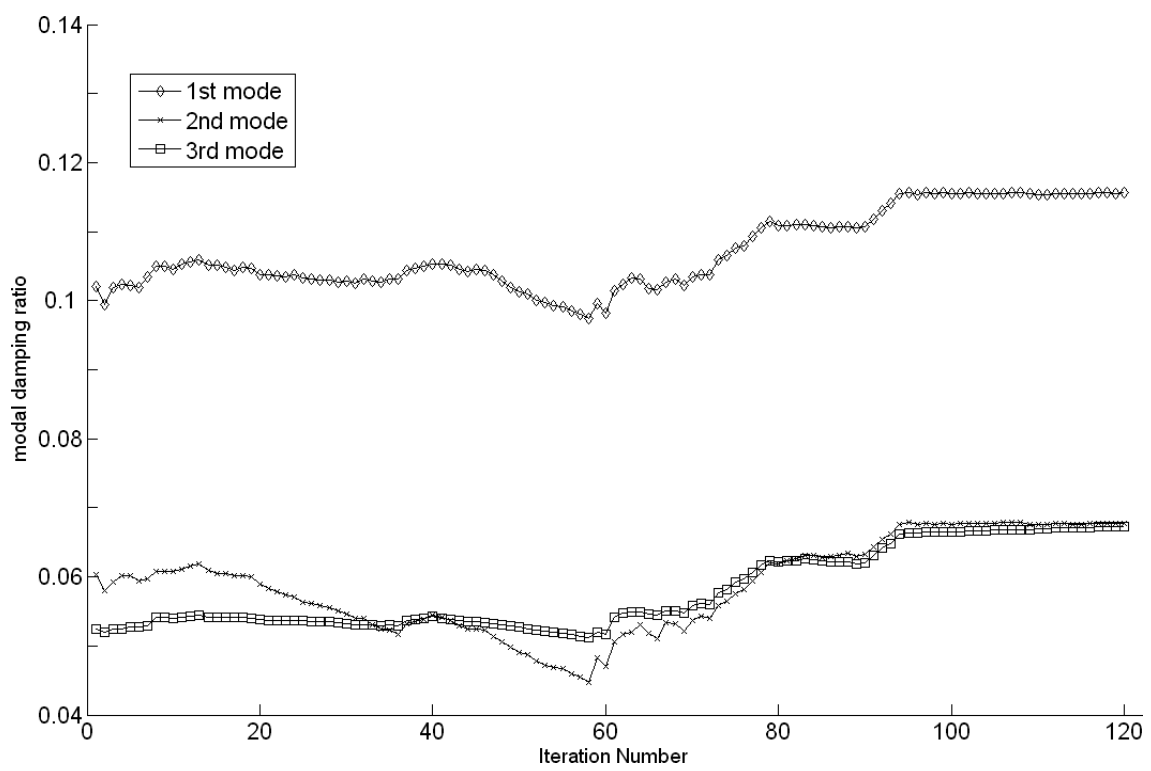

Fig. 25. Iteration histories for different modes $v f=0.8$.

of the displacement in the peak between $40 \%$ and $60 \%$ damping material applied is very small. The difference of the displacement in the peak between $80 \%$ and full covered by damping material is also very small. This means fully covered can be replaced by $80 \%$ covered or $60 \%$ covered can be replaced by $40 \%$ covered from the economic point of view.

\section{Conclusion}

In this paper, a finite element model to describe dynamic performances of plates with CLD treatments is developed on the basis of energy approach. The model is adopted together with an optimization algorithm in order to determine 


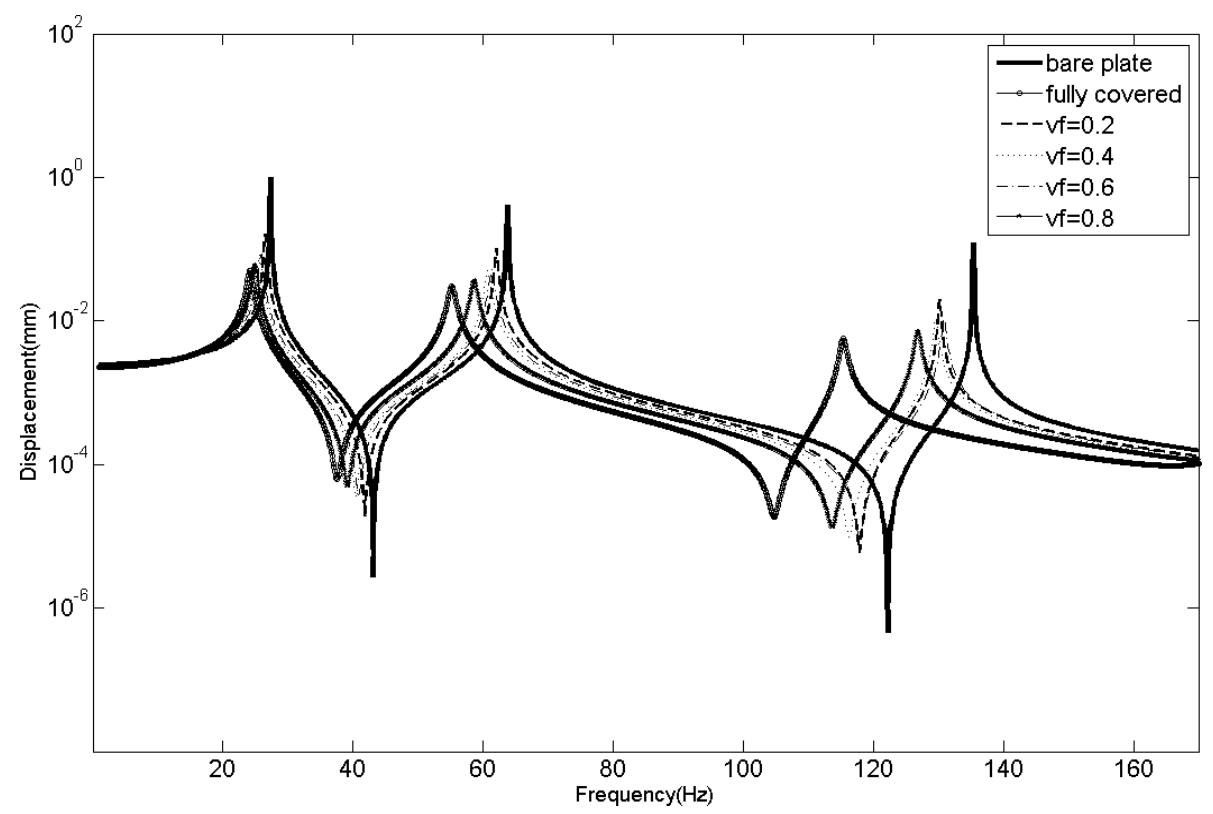

Fig. 26. Frequency response at the center of the plate excited by unit harmonic force at the same point.

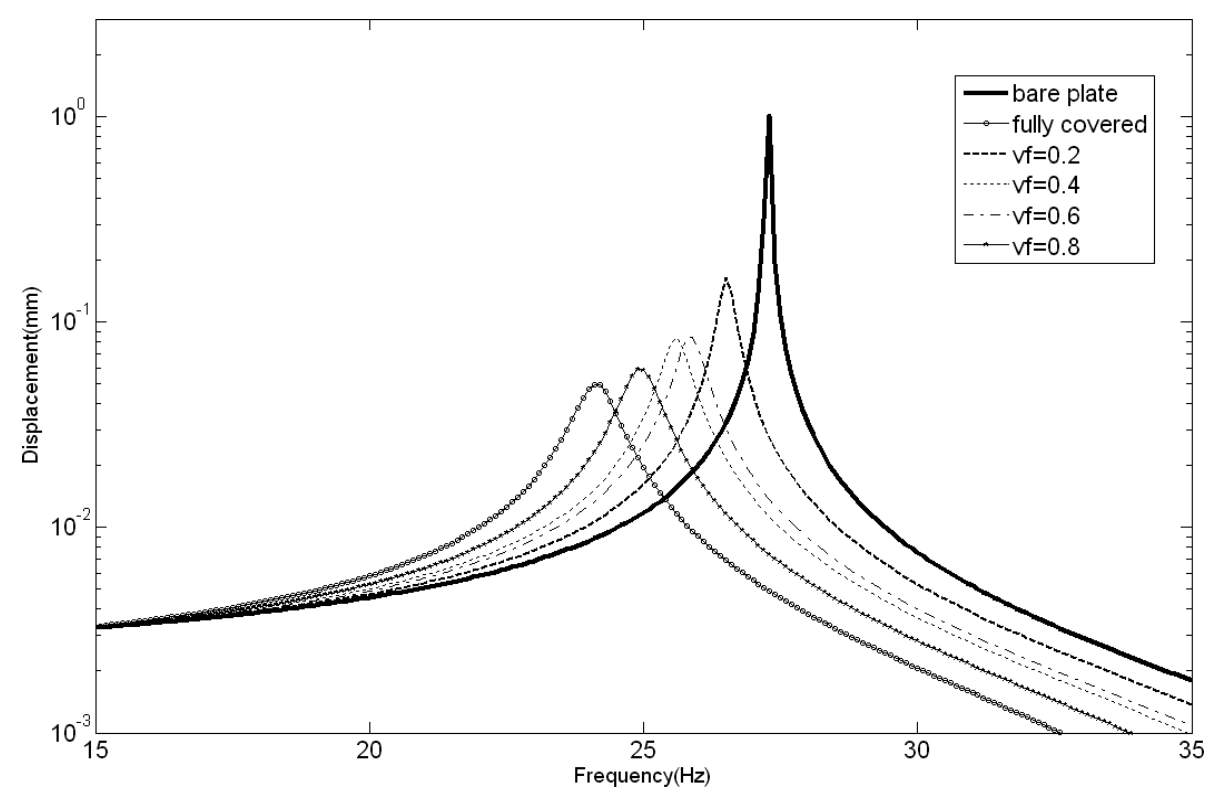

Fig. 27. The first natural frequency.

the optimal topologies of visco-elastic material distribution. The proposed approach is applied to the cantilever and simple supported plate/CLD system. In optimization process, the optimal topologies of visco-elastic material distribution are solved by Method of Moving Asymptote (MMA) approach which is modified so that it is available to solve the proposed optimization problems of visco-elastic material on plate/CLD systems. It is significant to note that the proposed approach results in a steady convergence process of modal damping ratios. Therefore, vibration energy dissipation can be enhanced. It is special to point that the optimal topologies of visco-elastic material distribution can be translated easily into realistic treatments for a real structure because the relative density of each visco-elastic material element can be transformed into variable thickness of each visco-elastic material element. This means that 


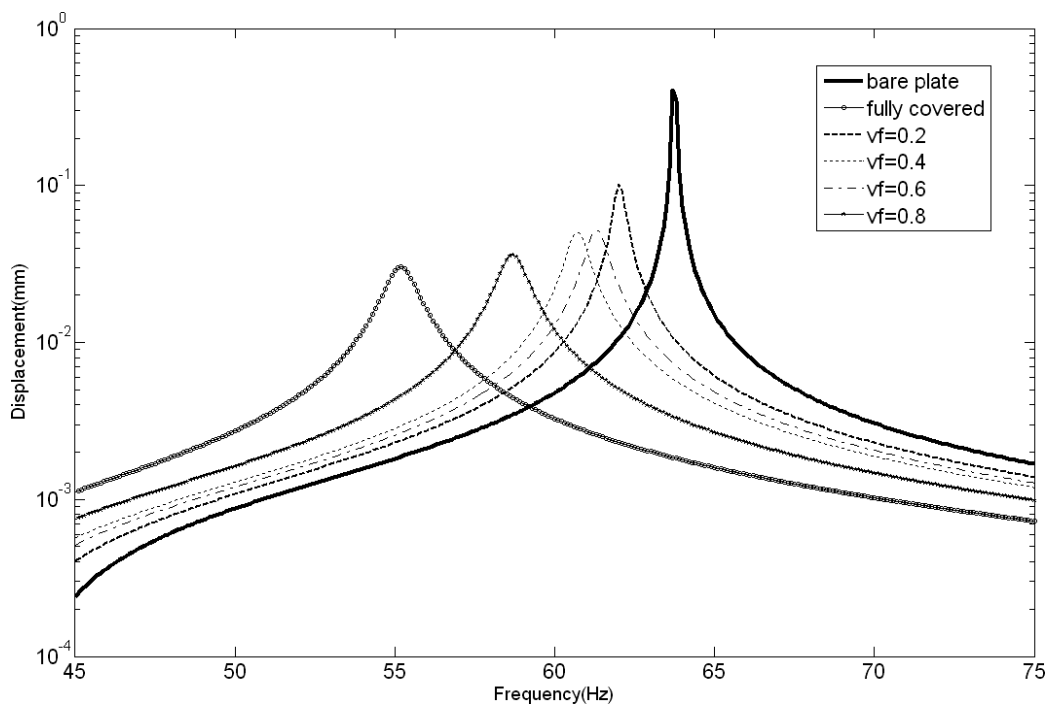

Fig. 28. The second natural frequency.

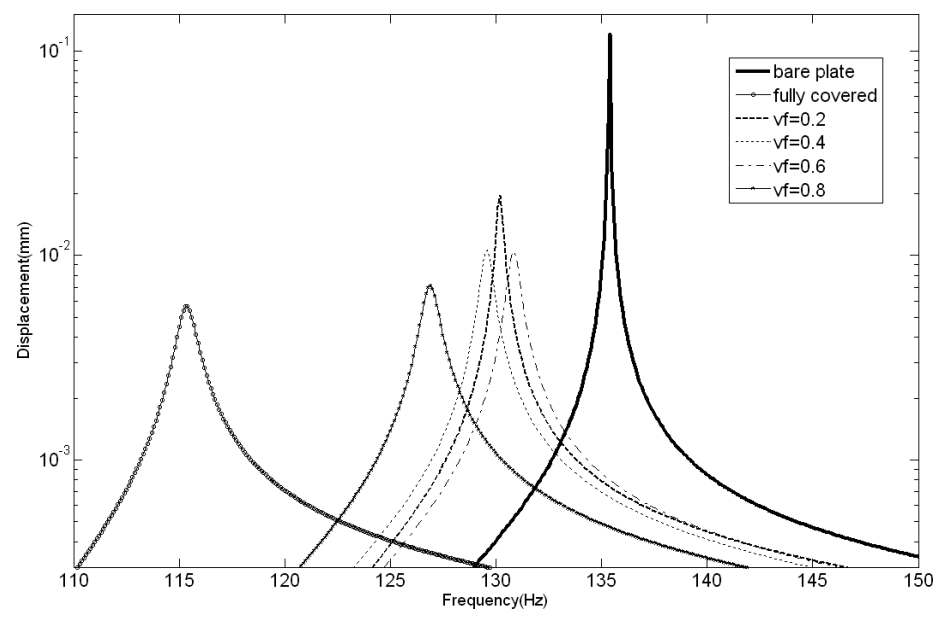

Fig. 29. The third natural frequency.

the final iteration results in a clear and real thickness modification for each element. The performance prediction of numerical examples identifies the effectiveness of the proposed topology optimization approach.

The proposed approach can be an important design tool for many structures with damping treatments in order to enhance energy dissipation and suppress structural vibration in an economical and effective means.

\section{Acknowledgements}

This work is funded by the Natural Science Foundation of China (Grant number 50775225). Special thanks are due to Prof. K. Svanberg of the Royal Institute of Technology in Stockholm for supplying the Method of Moving Asymptotes code. 


\section{Appendix}

\section{A. SPATIAL INTERPOLATING VECTORS}

The explicit forms of the spatial interpolating vectors, $\left[N_{1}\right],\left[N_{2}\right],\left[N_{3}\right],\left[N_{4}\right]$ and $\left[N_{5}\right]$, appearing in Eq. (7) are

$$
\left[N_{i}\right]=\left[\begin{array}{llll}
N_{i 1} & N_{i 2} & N_{i 3} & N_{i 4}
\end{array}\right](i=1,2,3,4,5)
$$

where

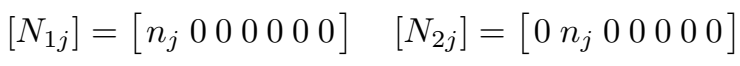

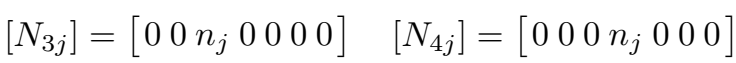

$$
\begin{aligned}
& {\left[N_{5 j}\right]=\left[\begin{array}{llllll}
0 & 0 & 0 & 0 & n_{w i} & n_{w i, x} \\
n_{w i, y}
\end{array}\right](j=1,2,3,4)}
\end{aligned}
$$

The shape functions at nodal location $\left\{x_{j}, y_{j}\right\}, n_{j}, n_{w j}, n_{w j, x}, n_{w j, y}$, can be expressed as

$$
\begin{aligned}
& n_{j}=\frac{1}{4}\left(1+\frac{x}{x_{j}}\right)\left(1+\frac{y}{y_{j}}\right) \quad n_{w j}=\frac{1}{8}\left(1+\frac{x}{x_{j}}\right)\left(1+\frac{y}{y_{j}}\right)\left[2+\frac{x}{x_{j}}\left(1-\frac{x}{x_{j}}\right)+\frac{y}{y_{j}}\left(1-\frac{y}{y_{j}}\right)\right] \\
& n_{w j, x}=-\frac{y_{j}}{8}\left(1+\frac{x}{x_{j}}\right)\left(1+\frac{y}{y_{j}}\right)^{2}\left(1-\frac{y}{y_{j}}\right) n_{w j, y}=\frac{x_{j}}{8}\left(1+\frac{x}{x_{j}}\right)^{2}\left(1+\frac{y}{y_{j}}\right)\left(1-\frac{x}{x_{j}}\right)
\end{aligned}
$$

The spatial interpolating vectors corresponding to $u_{v}, v_{v}, \beta_{x}$ and $\beta_{y}$ can be written as follows:

$$
\begin{aligned}
& {\left[N_{6}\right]=\frac{1}{2}\left[\left(\left[N_{1}\right]+\left[N_{3}\right]\right)+\frac{\delta_{c}-\delta_{p}}{2}\left[N_{5}\right]_{, x}\right] \quad\left[N_{7}\right]=\frac{1}{2}\left[\left(\left[N_{2}\right]+\left[N_{4}\right]\right)+\frac{\delta_{c}-\delta_{p}}{2}\left[N_{5}\right]_{, y}\right]} \\
& {\left[N_{8}\right]=\frac{1}{\delta_{v}}\left[\left(\left[N_{1}\right]-\left[N_{3}\right]\right)+\left(\frac{\delta_{c}+\delta_{p}}{2}+\delta_{v}\right)\left[N_{5}\right]_{, x}\right] \quad\left[N_{9}\right]=\frac{1}{\delta_{v}}\left[\left(\left[N_{2}\right]-\left[N_{4}\right]\right)+\left(\frac{\delta_{c}+\delta_{p}}{2}+\delta_{v}\right)\left[N_{5}\right]_{, y}\right]}
\end{aligned}
$$

\section{B. ELEMENTAL MATRICES}

In Eqs (8)-(14), the elemental mass matrices of each layer $\left[M_{p}^{(e)}\right],\left[M_{c}^{(e)}\right],\left[M_{v}^{(e)}\right]$ and the elemental stiffness matrices of each layer $\left[K_{p}^{(e)}\right],\left[K_{c}^{(e)}\right],\left[K_{v}^{(e)}\right],\left[K_{\beta v}^{(e)}\right]$ are defined as follows:

$$
\begin{aligned}
& {\left[M_{p}^{(e)}\right]=\rho_{p} \delta_{p} \int_{-a}^{a} \int_{-b}^{b}\left(\left[N_{3}\right]^{\mathbf{T}}\left[N_{3}\right]+\left[N_{4}\right]^{\mathbf{T}}\left[N_{4}\right]+\left[N_{5}\right]{ }_{\left.\left[N_{5}\right]\right)} \mathrm{d} x \mathrm{~d} y\right.} \\
& {\left[M_{c}^{(e)}\right]=\rho_{c} \delta_{c} \int_{-a}^{a} \int_{-b}^{b}\left(\left[N_{1}\right]^{\mathbf{T}}\left[N_{1}\right]+\left[N_{2}\right]{ }_{\left[N_{2}\right]+\left[N_{5}\right]}^{\mathbf{T}}\left[N_{5}\right]\right) \mathrm{d} x \mathrm{~d} y} \\
& {\left[M_{v}^{(e)}\right]=\rho_{v} \delta_{v} \int_{-a}^{a} \int_{-b}^{b}\left(\left[N_{6}\right]^{\mathbf{T}}\left[N_{6}\right]+\left[N_{7}\right]^{\mathbf{T}}\left[N_{7}\right]+\left[N_{5}\right]^{\mathbf{T}}\left[N_{5}\right]\right) \mathrm{d} x \mathrm{~d} y}
\end{aligned}
$$

and

$$
\begin{aligned}
& {\left[K_{p}^{(e)}\right]=\frac{E_{p} \delta_{p}}{1-\mu_{p}^{2}} \int_{-a}^{a} \int_{-b}^{b}\left[\begin{array}{l}
{\left[N_{3}\right]_{, x}} \\
{\left[N_{4}\right]_{, y}} \\
{\left[N_{3}\right]_{, y}+\left[N_{4}\right]_{, x}}
\end{array}\right]^{T}\left[\begin{array}{lll}
1 & \mu_{p} & 0 \\
\mu_{p} & 1 & 0 \\
0 & 0 & \frac{1-\mu_{p}}{2}
\end{array}\right]\left[\begin{array}{l}
{\left[N_{3}\right]_{, x}} \\
{\left[N_{4}\right]_{, y}} \\
{\left[N_{3}\right]_{, y}+\left[N_{4}\right]_{, x}}
\end{array}\right] d x d y+} \\
& \frac{E_{p} \delta_{p}^{3}}{12\left(1-\mu_{p}^{2}\right)} \int_{-a}^{a} \int_{-b}^{b}\left[\begin{array}{l}
{\left[N_{5}\right]_{, x x}} \\
{\left[N_{5}\right]_{, y y}} \\
2\left[N_{5}\right]_{, x y}
\end{array}\right]^{T}\left[\begin{array}{lll}
1 & \mu_{p} & 0 \\
\mu_{p} & 1 & 0 \\
0 & 0 & \frac{1-\mu_{p}}{2}
\end{array}\right]\left[\begin{array}{l}
{\left[N_{5}\right]_{, x x}} \\
{\left[N_{5}\right]_{, y y}} \\
2\left[N_{5}\right]_{, x y}
\end{array}\right] d x d y
\end{aligned}
$$




$$
\begin{aligned}
& {\left[K_{c}^{(e)}\right]=\frac{E_{c} \delta_{c}}{1-\mu_{c}^{2}} \int_{-a}^{a} \int_{-b}^{b}\left[\begin{array}{l}
{\left[N_{1}\right]_{, x}} \\
{\left[N_{2}\right]_{, y}} \\
{\left[N_{1}\right]_{, y}+\left[N_{2}\right]_{, x}}
\end{array}\right]^{T}\left[\begin{array}{lll}
1 & \mu_{c} & 0 \\
\mu_{c} & 1 & 0 \\
0 & 0 & \frac{1-\mu_{c}}{2}
\end{array}\right]\left[\begin{array}{l}
{\left[N_{1}\right]_{, x}} \\
{\left[N_{2}\right]_{, y}} \\
{\left[N_{1}\right]_{, y}+\left[N_{2}\right]_{, x}}
\end{array}\right] d x d y+} \\
& \frac{E_{c} \delta_{c}^{3}}{12\left(1-\mu_{c}^{2}\right)} \int_{-a}^{a} \int_{-b}^{b}\left[\begin{array}{l}
{\left[N_{5}\right]_{, x x}} \\
{\left[N_{5}\right]_{, y y}} \\
2\left[N_{5}\right]_{, x y}
\end{array}\right]^{T}\left[\begin{array}{lll}
1 & \mu_{c} & 0 \\
\mu_{c} & 1 & 0 \\
0 & 0 & \frac{1-\mu_{c}}{2}
\end{array}\right]\left[\begin{array}{l}
{\left[N_{5}\right]_{, x x}} \\
{\left[N_{5}\right]_{, y y}} \\
2\left[N_{5}\right]_{, x y}
\end{array}\right] d x d y \\
& {\left[K_{v}^{(e)}\right]=\frac{E_{v} \delta_{v}}{1-\mu_{v}^{2}} \int_{-a}^{a} \int_{-b}^{b}\left[\begin{array}{l}
{\left[N_{6}\right]_{, x}} \\
{\left[N_{7}\right]_{, y}} \\
{\left[N_{6}\right]_{, y}+\left[N_{7}\right]_{, x}}
\end{array}\right]^{T}\left[\begin{array}{lll}
1 & \mu_{v} & 0 \\
\mu_{v} & 1 & 0 \\
0 & 0 & \frac{1-\mu_{v}}{2}
\end{array}\right]\left[\begin{array}{l}
{\left[N_{6}\right]_{, x}} \\
{\left[N_{7}\right]_{, y}} \\
{\left[N_{6}\right]_{, y}+\left[N_{7}\right]_{, x}}
\end{array}\right] d x d y+} \\
& \frac{E_{v} \delta_{v}^{3}}{12\left(1-\mu_{v}^{2}\right)} \int_{-a}^{a} \int_{-b}^{b}\left[\begin{array}{l}
{\left[N_{5}\right]_{, x x}} \\
{\left[N_{5}\right]_{, y y}} \\
2\left[N_{5}\right]_{, x y}
\end{array}\right]^{T}\left[\begin{array}{lll}
1 & \mu_{v} & 0 \\
\mu_{v} & 1 & 0 \\
0 & 0 & \frac{1-\mu_{v}}{2}
\end{array}\right]\left[\begin{array}{l}
{\left[N_{5}\right]_{, x x}} \\
{\left[N_{5}\right]_{, y y}} \\
2\left[N_{5}\right]_{, x y}
\end{array}\right] d x d y \\
& {\left[K_{\beta v}^{(e)}\right]=G \delta_{v} \int_{-a}^{a} \int_{-b}^{b}\left(\left[N_{8}\right]^{\mathbf{T}}\left[N_{8}\right]+\left[N_{9}\right]^{\mathbf{T}}\left[N_{9}\right]\right) \mathrm{d} x \mathrm{~d} y}
\end{aligned}
$$

\section{References}

[1] A. Nashif, D. Jones and J. Henderson, Vibration Damping, J. Wiley and Sons, Inc., New York, 1985.

[2] E.M. Kerwin, Damping of Flexural Waves by a Constrained Viscoelastic Layer, Journal of the Acoustical Society of America 32(6) (1959), 952-962.

[3] R.A. DiTaranto and W. Blasingame, Composite Loss Factors of Selected Laminated Beams, Journal of the Acoustical Society of America 40(1) (1965), 187-194.

[4] R.A. DiTaranto, Theory of Vibratory Bending for Elastic and Viscoelastic Layered Finite Length Beams, ASME Journal of Applied Mechanics 32 (1965), 881-886.

[5] D.J. Mead and S. Markus, The Forced Vibration of a Three-Layered Damped Sandwich Beam with Arbitrary Boundary Conditions, Journal of Sound and Vibration 10(2) (1969), 163-175.

[6] K. Hedrih (Stevanivic) and J. Simonovic, Non-linear dynamics of the sandwich double circular plate system, International Journal of Non-Linear Mechanics, 2009, doi:10.1016/j.ijnonlinmec. 2009.12.007.

[7] R. Plunkett and C.T. Lee, Length Optimizatio n of Constrained Viscoelastic Layer Damping, Journal of the Acoustical Society of America 48(1) (1970), Part 2, 150-161.

[8] N. Alam and N.T. Asnani, Vibration and Damping Analysis of Multilayered Rectangular Plates with Constrained Viscoelastic Layers, Journal of Sound and Vibration 97(4) (1984), 597-614

[9] C.D. Johnson and D.A. Kienholz, Finite Element Prediction of Damping in Structures with Constrained Viscoelastic Layers, AIAA Journal 20(8) (1982), 1284-1290.

[10] R. Rikards, Finite Element Analysis of Vibration and Damping of Laminated Composites, Composite Structures 24 (1993), $193-204$.

[11] Y.C. Chen and S.C. Huang, An optimal placement of CLD treatment for vibration suppression of plates, International Journal of Mechanical Sciences 44 (2002), 1801-1821.

[12] H. Zheng, C. Cai and X.M. Tan, Optimization of partial constrained layer damping treatment for vibration energy minimization of vibrating beams, Computers and Structures 82 (2004), 2493-2507.

[13] H. Zheng, C. Cai, G.S.H. Pau and G.R. Liu, Minimizing vibration response of cylindrical shells through layout optimization of passive constrained layer damping treatments, Journal of Sound and Vibration 279 (2005), 739-756.

[14] M.P. Bendsoe and N. Kikuchi, Generating Optimal Topologies in Structural Design Using a Homogenization Method, Comp Mech in Appl Mech and Engrg 77 (1988), 197-224.

[15] M.P. Bendsøe and O. Sigmund, Topology optimization - theory, methods and applications. Springer, Berlin, 2003.

[16] M.P. Bendsøe, Optimization of structural topology, shape and material, Berlin, Heidelberg, New York: Springer 1995.

[17] R.E. Griffith and R.A. Stewart, A nonlinear programming technique for the optimization of continuous processing systems, Man Sci 7 (1961), 379-392.

[18] K. Svanberg, The method of moving asymptotes: a new method for structural optimization, International Journal for Numerical Method in Engineering 24 (1987), 359-373.

[19] K. Svanberg, A new globally convergent version of MMA. Technical Report TRITA-MAT-1999-OS2. Dept of Mathematics, KTH, Stockholm, 1999.

[20] M.P. Bendsøe and O. Sigmund, Material interpolations in topology optimization, Archive of Applied Mechanics 69 (1999), $635-654$. 
[21] D.Q. Yang, Topological Sensitivity Method for the Optimal Placement of Unconstrained Damping Materials under Dynamic Response Constraints, Journal of Shanghai Jiaotong University 37(7) (2003), 1109-1212.

[22] A. Mohammed, Al-Ajmi, Homogenization and structural topology optimization of constrained layer damping treatments. Ph.D Dissertation, University of Maryland, 2004.

[23] A. Baz and J. Ro, Vibration control of plates with active constrained layer damping, Smart Material Structures 5 (1996), 272-280

[24] L. Meirovitch, Elements of Vibration Analysis, McGraw-Hill, Inc, 1986.

[25] M.P. Bendsoe, Optimal shape design as a material distribution problem, Struct Optim 1 (1989), 193-202.

[26] M. Zhou and G.I.N. Rozvany, The COC algorithm, partII: Topological, geometry and generalized shape optimization, Comp Meth Appl Mech Engrng 89 (1991), 197-224. 

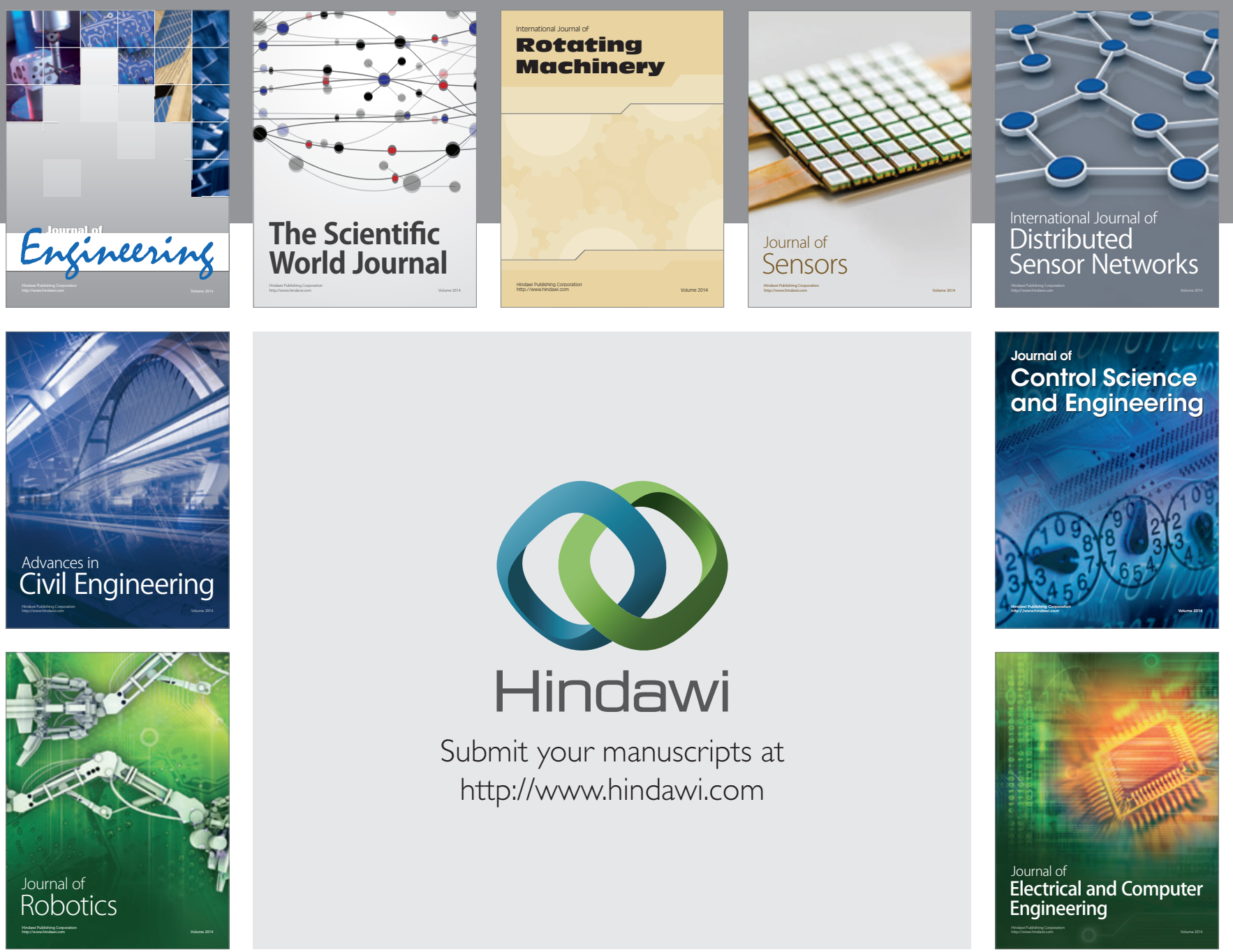

Submit your manuscripts at

http://www.hindawi.com
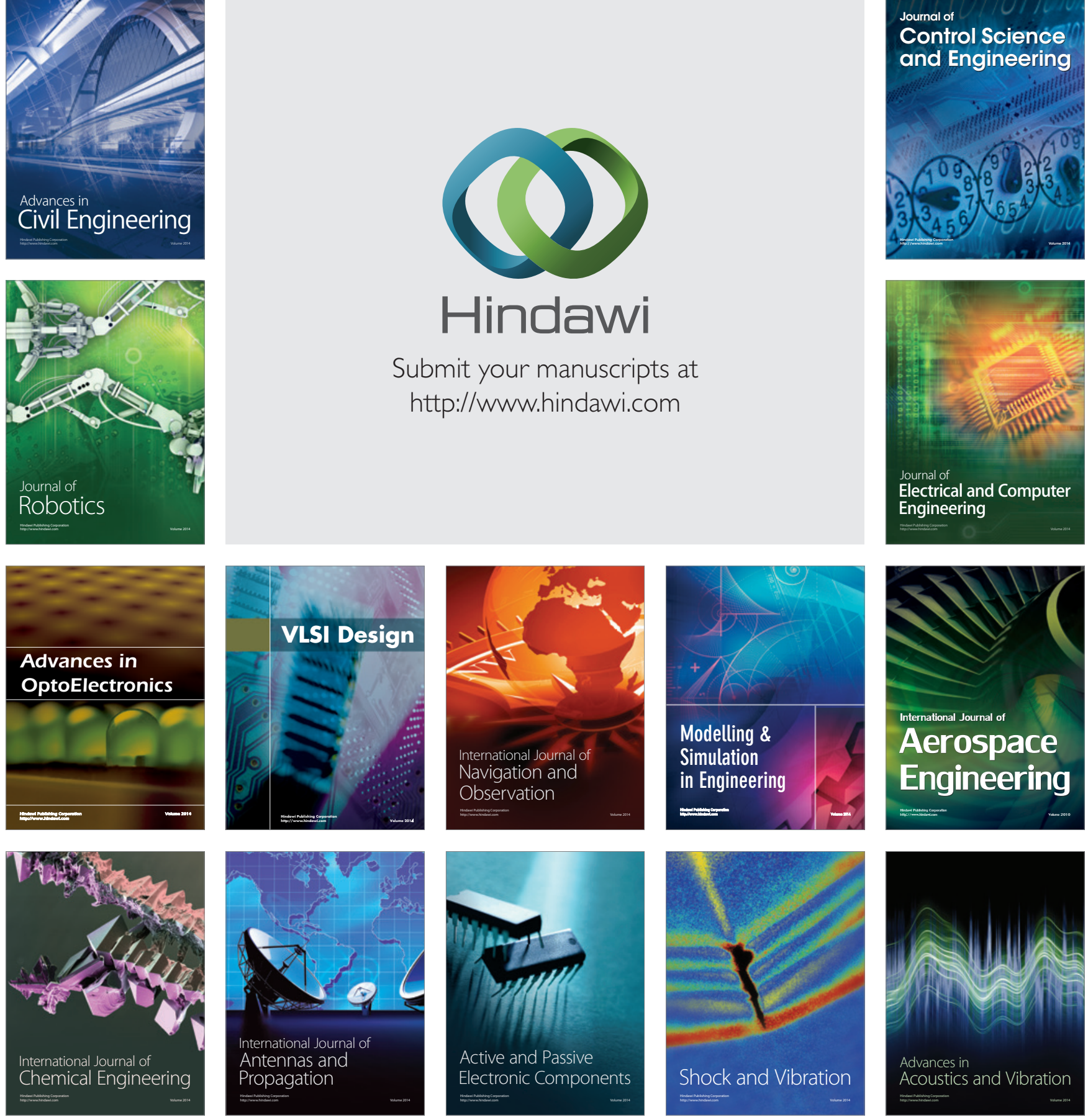